\title{
Learning with Hierarchical-Deep Models
}

\author{
Ruslan Salakhutdinov, Joshua B. Tenenbaum, and Antonio Torralba, Member, IEEE
}

\begin{abstract}
We introduce HD (or "Hierarchical-Deep") models, a new compositional learning architecture that integrates deep learning models with structured hierarchical Bayesian (HB) models. Specifically, we show how we can learn a hierarchical Dirichlet process (HDP) prior over the activities of the top-level features in a deep Boltzmann machine (DBM). This compound HDP-DBM model learns to learn novel concepts from very few training example by learning low-level generic features, high-level features that capture correlations among low-level features, and a category hierarchy for sharing priors over the high-level features that are typical of different kinds of concepts. We present efficient learning and inference algorithms for the HDP-DBM model and show that it is able to learn new concepts from very few examples on CIFAR-100 object recognition, handwritten character recognition, and human motion capture datasets.
\end{abstract}

Index Terms-Deep networks, deep Boltzmann machines, hierarchical Bayesian models, one-shot learning

\section{INTRODUCTION}

$\mathrm{T}$ HE ability to learn abstract representations that support transfer to novel but related tasks lies at the core of many problems in computer vision, natural language processing, cognitive science, and machine learning. In typical applications of machine classification algorithms today, learning a new concept requires tens, hundreds, or thousands of training examples. For human learners, however, just one or a few examples are often sufficient to grasp a new category and make meaningful generalizations to novel instances [15], [25], [31], [44]. Clearly, this requires very strong but also appropriately tuned inductive biases. The architecture we describe here takes a step toward this ability by learning several forms of abstract knowledge at different levels of abstraction that support transfer of useful inductive biases from previously learned concepts to novel ones.

We call our architectures compound HD models, where "HD" stands for "Hierarchical-Deep," because they are derived by composing hierarchical nonparametric Bayesian models with deep networks, two influential approaches from the recent unsupervised learning literature with complementary strengths. Recently introduced deep learning models, including deep belief networks (DBNs) [12], deep Boltzmann machines (DBM) [29], deep autoencoders [19], and many others [9], [10], [21], [22], [26], [32], [34], [43], have been shown to learn useful distributed feature

- R. Salakhutdinov is with the Department of Statistics and Computer Science, University of Toronto, Toronto, ON M5S 3G3, Canada. E-mail: rsalakhu@utstat.toronto.edu.

- J.B. Tenenbaum is with the Department of Brain and Cognitive Sciences, Massachusetts Institute of Technology, Cambridge, MA 02139.

E-mail: jbt@mit.edu.

- A. Torralba is with the Computer Science and Artificial Intelligence Laboratory, Massachusetts Institute of Technology, Cambridge, MA 02139.E-mail: torralba@mit.edu.

Manuscript received 18 Apr. 2012; revised 30 Aug. 2012; accepted 30 Nov. 2012; published online 19 Dec. 2012.

Recommended for acceptance by $M$. Welling.

For information on obtaining reprints of this article, please send e-mail to: tpami@computer.org, and reference IEEECS Log Number

TPAMI-2012-04-0302.

Digital Object Identifier no. 10.1109/TPAMI.2012.269. representations for many high-dimensional datasets. The ability to automatically learn in multiple layers allows deep models to construct sophisticated domain-specific features without the need to rely on precise human-crafted input representations, increasingly important with the proliferation of datasets and application domains.

While the features learned by deep models can enable more rapid and accurate classification learning, deep networks themselves are not well suited to learning novel classes from few examples. All units and parameters at all levels of the network are engaged in representing any given input ("distributed representations"), and are adjusted together during learning. In contrast, we argue that learning new classes from a handful of training examples will be easier in architectures that can explicitly identify only a small number of degrees of freedom (latent variables and parameters) that are relevant to the new concept being learned, and thereby achieve more appropriate and flexible transfer of learned representations to new tasks. This ability is the hallmark of hierarchical Bayesian (HB) models, recently proposed in computer vision, statistics, and cognitive science [8], [11], [15], [28], [44] for learning from few examples. Unlike deep networks, these HB models explicitly represent category hierarchies that admit sharing the appropriate abstract knowledge about the new class's parameters via a prior abstracted from related classes. HB approaches, however, have complementary weaknesses relative to deep networks. They typically rely on domainspecific hand-crafted features [2], [11] (e.g., GIST, SIFT features in computer vision, MFCC features in speech perception domains). Committing to the a-priori defined feature representations, instead of learning them from data, can be detrimental. This is especially important when learning complex tasks, as it is often difficult to hand-craft high-level features explicitly in terms of raw sensory input. Moreover, many $\mathrm{HB}$ approaches often assume a fixed hierarchy for sharing parameters [6], [33] instead of discovering how parameters are shared among classes in an unsupervised fashion.

In this paper, we propose compound HD architectures that integrate these deep models with structured HB 
models. In particular, we show how we can learn a hierarchical Dirichlet process (HDP) prior over the activities of the top-level features in a DBM, coming to represent both a layered hierarchy of increasingly abstract features and a tree-structured hierarchy of classes. Our model depends minimally on domain-specific representations and achieves state-of-the-art performance by unsupervised discovery of three components: 1) low-level features that abstract from the raw high-dimensional sensory input (e.g., pixels, or three-dimensional joint angles) and provide a useful first representation for all concepts in a given domain; 2) highlevel part-like features that express the distinctive perceptual structure of a specific class, in terms of class-specific correlations over low-level features; and 3) a hierarchy of superclasses for sharing abstract knowledge among related classes via a prior on which higher level features are likely to be distinctive for classes of a certain kind and are thus likely to support learning new concepts of that kind.

We evaluate the compound HDP-DBM model on three different perceptual domains. We also illustrate the advantages of having a full generative model, extending from highly abstract concepts all the way down to sensory inputs: We cannot only generalize class labels but also synthesize new examples in novel classes that look reasonably natural, and we can significantly improve classification performance by learning parameters at all levels jointly by maximizing a joint log-probability score.

There have also been several approaches in the computer vision community addressing the problem of learning with few examples. Torralba et al. [42] proposed using several boosted detectors in a multitask setting, where features are shared between several categories. Bart and Ullman [3] further proposed a cross-generalization framework for learning with few examples. Their key assumption is that new features for a novel category are selected from the pool of features that was useful for previously learned classification tasks. In contrast to our work, the above approaches are discriminative by nature and do not attempt to identify similar or relevant categories. Babenko et al. [1] used a boosting approach that simultaneously groups together categories into several supercategories, sharing a similarity metric within these classes. They, however, did not attempt to address transfer learning problem, and primarily focused on large-scale image retrieval tasks. Finally, Fei-Fei et al. [11] used an HB approach, with a prior on the parameters of new categories that was induced from other categories. However, their approach was not ideal as a generic approach to transfer learning with few examples. They learned only a single prior shared across all categories. The prior was learned from only three categories, chosen by hand. Compared to our work, they used a more elaborate visual object model, based on multiple parts with separate appearance and shape components.

\section{Deep Boltzmann Machines}

A DBM is a network of symmetrically coupled stochastic binary units. It contains a set of visible units $\mathbf{v} \in\{0,1\}^{D}$, and a sequence of layers of hidden units $\mathbf{h}^{(1)} \in\{0,1\}^{F_{1}}$, $\mathbf{h}^{(2)} \in\{0,1\}^{F_{2}} \ldots \mathbf{h}^{(L)} \in\{0,1\}^{F_{L}}$. There are connections only between hidden units in adjacent layers, as well as between visible and hidden units in the first hidden layer. Consider a
DBM with three hidden layers ${ }^{1}$ (i.e., $L=3$ ). The energy of the joint configuration $\{\mathbf{v}, \mathbf{h}\}$ is defined as

$$
\begin{aligned}
E(\mathbf{v}, \mathbf{h} ; \boldsymbol{\psi})= & -\sum_{i j} W_{i j}^{(1)} v_{i} h_{j}^{(1)}-\sum_{j l} W_{j l}^{(2)} h_{j}^{(1)} h_{l}^{(2)} \\
& -\sum_{l k} W_{l k}^{(3)} h_{l}^{(2)} h_{k}^{(3)}
\end{aligned}
$$

where $\mathbf{h}=\left\{\mathbf{h}^{(1)}, \mathbf{h}^{(2)}, \mathbf{h}^{(3)}\right\}$ represent the set of hidden units and $\boldsymbol{\psi}=\left\{\mathbf{W}^{(1)}, \mathbf{W}^{(2)}, \mathbf{W}^{(3)}\right\}$ are the model parameters, representing visible-to-hidden and hidden-to-hidden symmetric interaction terms. ${ }^{2}$

The probability that the model assigns to a visible vector $\mathbf{v}$ is given by the Boltzmann distribution:

$$
P(\mathbf{v} ; \boldsymbol{\psi})=\frac{1}{\mathcal{Z}(\boldsymbol{\psi})} \sum_{\mathbf{h}} \exp \left(-E\left(\mathbf{v}, \mathbf{h}^{(1)}, \mathbf{h}^{(2)}, \mathbf{h}^{(3)} ; \boldsymbol{\psi}\right)\right) .
$$

Observe that setting both $\mathbf{W}^{(2)}=0$ and $\mathbf{W}^{(3)}=0$ recovers the simpler Restricted Boltzmann Machine (RBM) model.

The conditional distributions over the visible and the three sets of hidden units are given by

$$
\begin{aligned}
p\left(h_{j}^{(1)}=1 \mid \mathbf{v}, \mathbf{h}^{(2)}\right) & =g\left(\sum_{i=1}^{D} W_{i j}^{(1)} v_{i}+\sum_{l=1}^{F_{2}} W_{j l}^{(2)} h_{l}^{(2)}\right), \\
p\left(h_{l}^{(2)}=1 \mid \mathbf{h}^{(1)}, \mathbf{h}^{(3)}\right) & =g\left(\sum_{j=1}^{F_{1}} W_{j l}^{(2)} h_{j}^{(1)}+\sum_{k=1}^{F_{3}} W_{l k}^{(3)} h_{k}^{(3)}\right), \\
p\left(h_{k}^{(3)}=1 \mid \mathbf{h}^{(2)}\right) & =g\left(\sum_{l=1}^{F_{2}} W_{l k}^{(3)} h_{l}^{(2)}\right), \\
p\left(v_{i}=1 \mid \mathbf{h}^{(1)}\right) & =g\left(\sum_{j=1}^{F_{1}} W_{i j}^{(1)} h_{j}^{(1)}\right),
\end{aligned}
$$

where $g(x)=1 /(1+\exp (-x))$ is the logistic function.

The derivative of the log-likelihood with respect to the model parameters $\boldsymbol{\psi}$ can be obtained from (1):

$$
\begin{aligned}
& \frac{\partial \log P(\mathbf{v} ; \boldsymbol{\psi})}{\partial \mathbf{W}^{(1)}}=\mathrm{E}_{P_{\text {data }}}\left[\mathbf{v h}^{(1)^{\top}}\right]-\mathrm{E}_{P_{\text {model }}}\left[\mathbf{v h}^{(1)^{\top}}\right], \\
& \frac{\partial \log P(\mathbf{v} ; \boldsymbol{\psi})}{\partial \mathbf{W}^{(2)}}=\mathrm{E}_{P_{\text {data }}}\left[\mathbf{h}^{(1)} \mathbf{h}^{(2)^{\top}}\right]-\mathrm{E}_{P_{\text {model }}}\left[\mathbf{h}^{(1)} \mathbf{h}^{(2)^{\top}}\right], \\
& \frac{\partial \log P(\mathbf{v} ; \boldsymbol{\psi})}{\partial \mathbf{W}^{(3)}}=\mathrm{E}_{P_{\text {data }}}\left[\mathbf{h}^{(2)} \mathbf{h}^{(3)^{\top}}\right]-\mathrm{E}_{P_{\text {model }}}\left[\mathbf{h}^{(2)} \mathbf{h}^{(3)^{\top}}\right],
\end{aligned}
$$

where $\mathrm{E}_{P_{\text {data }}}[\cdot]$ denotes an expectation with respect to the completed data distribution:

$$
P_{\text {data }}(\mathbf{h}, \mathbf{v} ; \boldsymbol{\psi})=P(\mathbf{h} \mid \mathbf{v} ; \boldsymbol{\psi}) P_{\text {data }}(\mathbf{v}),
$$

with $P_{\text {data }}(\mathbf{v})=\frac{1}{N} \sum_{n} \delta_{\mathbf{v}_{n}}$ representing the empirical distribution and $\mathrm{E}_{P_{\text {model }}}[\cdot]$ is an expectation with respect to the distribution defined by the model (1). We will sometimes refer to $\mathrm{E}_{P_{\text {data }}}[\cdot]$ as the data-dependent expectation and $\mathrm{E}_{P_{\text {model }}}[$. as the model's expectation.

Exact maximum likelihood learning in this model is intractable. The exact computation of the data-dependent expectation takes time that is exponential in the number of

1. For clarity, we use three hidden layers. Extensions to models with more than three layers is trivial.

2. We have omitted the bias terms for clarity of presentation. Biases are equivalent to weights on a connection to a unit whose state is fixed at 1 . 
hidden units, whereas the exact computation of the models expectation takes time that is exponential in the number of hidden and visible units.

\subsection{Approximate Learning}

The original learning algorithm for Boltzmann machines used randomly initialized Markov chains to approximate both expectations to estimate gradients of the likelihood function [14]. However, this learning procedure is too slow to be practical. Recently, Salakhutdinov and Hinton [29] proposed a variational approach, where mean-field inference is used to estimate data-dependent expectations and an MCMC-based stochastic approximation procedure is used to approximate the models expected sufficient statistics.

\subsubsection{A Variational Approach to Estimating the Data-Dependent Statistics}

Consider any approximating distribution $Q(\mathbf{h} \mid \mathbf{v} ; \boldsymbol{\mu})$, parametarized by a vector of parameters $\boldsymbol{\mu}$, for the posterior $P(\mathbf{h} \mid \mathbf{v} ; \boldsymbol{\psi})$. Then the log-likelihood of the DBM model has the following variational lower bound:

$$
\begin{aligned}
\log P(\mathbf{v} ; \boldsymbol{\psi}) & \geq \sum_{\mathbf{h}} Q(\mathbf{h} \mid \mathbf{v} ; \boldsymbol{\mu}) \log P(\mathbf{v}, \mathbf{h} ; \boldsymbol{\psi})+\mathcal{H}(Q) \\
& \geq \log P(\mathbf{v} ; \boldsymbol{\psi})-\mathrm{KL}(Q(\mathbf{h} \mid \mathbf{v} ; \boldsymbol{\mu}) \| P(\mathbf{h} \mid \mathbf{v} ; \boldsymbol{\psi})),
\end{aligned}
$$

where $\mathcal{H}(\cdot)$ is the entropy functional and $\operatorname{KL}(Q \| P)$ denotes the Kullback-Leibler divergence between the two distributions. The bound becomes tight if and only if $Q(\mathbf{h} \mid \mathbf{v} ; \boldsymbol{\mu})=$ $P(\mathbf{h} \mid \mathbf{v} ; \boldsymbol{\psi})$.

Variational learning has the nice property that in addition to maximizing the log-likelihood of the data, it also attempts to find parameters that minimize the Kullback-Leibler divergence between the approximating and true posteriors.

For simplicity and speed, we approximate the true posterior $P(\mathbf{h} \mid \mathbf{v} ; \boldsymbol{\psi})$ with a fully factorized approximating distribution over the three sets of hidden units, which corresponds to so-called mean-field approximation:

$$
Q^{M F}(\mathbf{h} \mid \mathbf{v} ; \boldsymbol{\mu})=\prod_{j=1}^{F_{1}} \prod_{l=1}^{F_{2}} \prod_{k=1}^{F_{3}} q\left(h_{j}^{(1)} \mid \mathbf{v}\right) q\left(h_{l}^{(2)} \mid \mathbf{v}\right) q\left(h_{k}^{(3)} \mid \mathbf{v}\right),
$$

where $\boldsymbol{\mu}=\left\{\boldsymbol{\mu}^{(1)}, \boldsymbol{\mu}^{(2)}, \boldsymbol{\mu}^{(3)}\right\}$ are the mean-field parameters with $q\left(h_{i}^{(l)}=1\right)=\mu_{i}^{(l)}$ for $l=1,2,3$. In this case, the variational lower bound on the log-probability of the data takes a particularly simple form:

$$
\begin{aligned}
\log P(\mathbf{v} ; \boldsymbol{\psi}) \geq & \sum_{\mathbf{h}} Q^{M F}(\mathbf{h} \mid \mathbf{v} ; \boldsymbol{\mu}) \log P(\mathbf{v}, \mathbf{h} ; \boldsymbol{\psi})+\mathcal{H}\left(Q^{M F}\right) \\
\geq & \mathbf{v}^{\top} \mathbf{W}^{(1)} \boldsymbol{\mu}^{(1)}+\boldsymbol{\mu}^{(1)^{\top}} \mathbf{W}^{(2)} \boldsymbol{\mu}^{(2)}+ \\
& +\boldsymbol{\mu}^{(2)^{\top}} \mathbf{W}^{(3)} \boldsymbol{\mu}^{(3)}-\log \mathcal{Z}(\boldsymbol{\psi})+\mathcal{H}\left(Q^{M F}\right) .
\end{aligned}
$$

Learning proceeds as follows: For each training example, we maximize this lower bound with respect to the variational parameters $\boldsymbol{\mu}$ for fixed parameters $\boldsymbol{\psi}$, which results in the mean-field fixed-point equations:

$$
\mu_{j}^{(1)} \leftarrow g\left(\sum_{i=1}^{D} W_{i j}^{(1)} v_{i}+\sum_{l=1}^{F_{2}} W_{j l}^{(2)} \mu_{l}^{(2)}\right),
$$

Algorithm 1. Learning Procedure for a Deep Boltzmann Machine with Three Hidden Layers.

1: Given: a training set of $N$ binary data vectors $\{\mathbf{v}\}_{n=1}^{N}$, and $M$, the number of persistent Markov chains (i.e., particles).

2: Randomly initialize parameter vector $\boldsymbol{\psi}_{0}$ and $M$ samples: $\left\{\tilde{\mathbf{v}}_{0,1}, \tilde{\mathbf{h}}_{0,1}\right\} \ldots\left\{\tilde{\mathbf{v}}_{0, M}, \tilde{\mathbf{h}}_{0, M}\right\}$, where $\tilde{\mathbf{h}}=\left\{\tilde{\mathbf{h}}^{(1)}, \tilde{\mathbf{h}}^{(2)}, \tilde{\mathbf{h}}^{(3)}\right\}$.

3: for $t=0$ to $T$ (number of iterations) do

4: // Variational Inference:

5: $\quad$ for each training example $\mathbf{v}_{n}, n=1$ to $N$ do

6: $\quad$ Randomly initialize $\boldsymbol{\mu}=\left\{\boldsymbol{\mu}^{(1)}, \boldsymbol{\mu}^{(2)}, \boldsymbol{\mu}^{(3)}\right\}$ and run mean-field updates until convergence, using (7), (8), (9).

7: $\quad$ Set $\boldsymbol{\mu}_{n}=\boldsymbol{\mu}$.

8: end for

9: // Stochastic Approximation:

10: for each sample $m=1$ to $M$ (number of persistent Markov chains) do

11: $\quad$ Sample $\left(\tilde{\mathbf{v}}_{t+1, m}, \tilde{\mathbf{h}}_{t+1, m}\right)$ given $\left(\tilde{\mathbf{v}}_{t, m}, \tilde{\mathbf{h}}_{t, m}\right)$ by running a Gibbs sampler for one step (2).

12: end for

13: // Parameter Update:

14: $\mathbf{W}_{t+1}^{(1)}=\mathbf{W}_{t}^{(1)}+\alpha_{t}\left(\frac{1}{N} \sum_{n=1}^{N} \mathbf{v}_{n}\left(\boldsymbol{\mu}_{n}^{(1)}\right)^{\top}-\right.$

$$
\left.\frac{1}{M} \sum_{m=1}^{M} \tilde{\mathbf{v}}_{t+1, m}\left(\tilde{\mathbf{h}}_{t+1, m}^{(1)}\right)^{\top}\right) .
$$

15: $\quad \mathbf{W}_{t+1}^{(2)}=\mathbf{W}_{t}^{(2)}+\alpha_{t}\left(\frac{1}{N} \sum_{n=1}^{N} \boldsymbol{\mu}_{n}^{(1)}\left(\boldsymbol{\mu}_{n}^{(2)}\right)^{\top}-\right.$

$$
\left.\frac{1}{M} \sum_{m=1}^{M} \tilde{\mathbf{h}}_{t+1, m}^{(1)}\left(\tilde{\mathbf{h}}_{t+1, m}^{(2)}\right)^{\top}\right) .
$$

16: $\quad \mathbf{W}_{t+1}^{(3)}=\mathbf{W}_{t}^{(3)}+\alpha_{t}\left(\frac{1}{N} \sum_{n=1}^{N} \boldsymbol{\mu}_{n}^{(2)}\left(\boldsymbol{\mu}_{n}^{(3)}\right)^{\top}-\right.$

17: Decrease $\alpha_{t}$.

$$
\left.\frac{1}{M} \sum_{m=1}^{M} \tilde{\mathbf{h}}_{t+1, m}^{(2)}\left(\tilde{\mathbf{h}}_{t+1, m}^{(3)}\right)^{\top}\right) .
$$

18: end for

$$
\begin{gathered}
\mu_{l}^{(2)} \leftarrow g\left(\sum_{j=1}^{F_{1}} W_{j l}^{(2)} \mu_{j}^{(1)}+\sum_{k=1}^{F_{3}} W_{l k}^{(3)} \mu_{k}^{(3)}\right), \\
\mu_{k}^{(3)} \leftarrow g\left(\sum_{l=1}^{F_{2}} W_{l k}^{(3)} \mu_{l}^{(2)}\right),
\end{gathered}
$$

where $g(x)=1 /(1+\exp (-x))$ is the logistic function. To solve these fixed-point equations, we simply cycle through layers, updating the mean-field parameters within a single layer. Note the close connection between the form of the mean-field fixed point updates and the form of the conditional distribution ${ }^{3}$ defined by (2).

\subsubsection{A Stochastic Approximation Approach for Estimating the Data-Independent Statistics}

Given the variational parameters $\boldsymbol{\mu}$, the model parameters $\boldsymbol{\psi}$ are then updated to maximize the variational bound using an MCMC-based stochastic approximation [29], [39], [46].

3. Implementing the mean-field requires no extra work beyond implementing the Gibbs sampler. 
Learning with stochastic approximation is straightforward. Let $\boldsymbol{\psi}_{t}$ and $\mathbf{x}_{t}=\left\{\mathbf{v}_{t}, \mathbf{h}_{t}^{(1)}, \mathbf{h}_{t}^{(2)}, \mathbf{h}_{t}^{(3)}\right\}$ be the current parameters and the state. Then $\mathbf{x}_{t}$ and $\boldsymbol{\psi}_{t}$ are updated sequentially as follows:

- Given $\mathbf{x}_{t}$, sample a new state $\mathbf{x}_{t+1}$ from the transition operator $T_{\boldsymbol{\psi}_{t}}\left(\mathbf{x}_{t+1} \leftarrow \mathbf{x}_{t}\right)$ that leaves $P\left(\cdot ; \boldsymbol{\psi}_{t}\right)$ invariant. This can be accomplished by using Gibbs sampling (see (2)).

- A new parameter $\boldsymbol{\psi}_{t+1}$ is then obtained by making a gradient step, where the intractable model's expectation $\mathrm{E}_{P_{\text {model }}}[\cdot]$ in the gradient is replaced by a point estimate at sample $\mathbf{x}_{t+1}$.

In practice, we typically maintain a set of $M$ "persistent" sample particles $X_{t}=\left\{\mathbf{x}_{t, 1}, \ldots, \mathbf{x}_{t, M}\right\}$, and use an average over those particles. The overall learning procedure for DBMs is summarized in Algorithm 1.

Stochastic approximation provides asymptotic convergence guarantees and belongs to the general class of Robbins-Monro approximation algorithms [27], [46]. Precise sufficient conditions that ensure almost sure convergence to an asymptotically stable point are given in [45], [46], and [47]. One necessary condition requires the learning rate to decrease with time so that $\sum_{t=0}^{\infty} \alpha_{t}=\infty$ and $\sum_{t=0}^{\infty} \alpha_{t}^{2}<\infty$. This condition can, for example, be satisfied simply by setting $\alpha_{t}=a /(b+t)$, for positive constants $a>0, b>0$. Other conditions ensure that the speed of convergence of the Markov chain, governed by the transition operator $T_{\psi}$, does not decrease too fast as $\boldsymbol{\psi}$ tends to infinity. Typically, in practice the sequence $\left|\boldsymbol{\psi}^{t}\right|$ is bounded, and the Markov chain, governed by the transition kernel $T_{\boldsymbol{\psi}}$, is ergodic. Together with the condition on the learning rate, this ensures almost sure convergence of the stochastic approximation algorithm to an asymptotically stable point.

\subsubsection{Greedy Layerwise Pretraining of DBMs}

The learning procedure for DBMs described above can be used by starting with randomly initialized weights, but it works much better if the weights are initialized sensibly. We therefore use a greedy layerwise pretraining strategy by learning a stack of modified RBMs (for details see [29]).

This pretraining procedure is quite similar to the pretraining procedure of DBNs [12], and it allows us to perform approximate inference by a single bottom-up pass. This fast approximate inference is then used to initialize the mean-field, which then converges much faster than meanfield with random initialization.

\subsection{Gaussian-Bernoulli DBMs}

We now briefly describe a Gaussian-Bernoulli DBM model, which we will use to model real-valued data, such as images of natural scenes and motion capture data. Gaussian-Bernoulli DBMs represent a generalization of a simpler class of models, called Gaussian-Bernoulli RBMs, which have been successfully applied to various tasks, including image classification, video action recognition, and speech recognition [17], [20], [23], [35].

In particular, consider modeling visible real-valued units $\mathbf{v} \in \mathbb{R}^{D}$ and let $\mathbf{h}^{(1)} \in\{0,1\}^{F_{1}}, \mathbf{h}^{(2)} \in\{0,1\}^{F_{2}}$, and $\mathbf{h}^{(3)} \in$

4. The code for pretraining and generative learning of the DBM model is available at http://www.utstat.toronto.edu/ rsalakhu/DBM.html.
$\{0,1\}^{F_{3}}$ be binary stochastic hidden units. The energy of the joint configuration $\left\{\mathbf{v}, \mathbf{h}^{(1)}, \mathbf{h}^{(2)}, \mathbf{h}^{(3)}\right\}$ of the three-hiddenlayer Gaussian-Bernoulli DBM is defined as follows:

$$
\begin{aligned}
E(\mathbf{v}, \mathbf{h} ; \boldsymbol{\psi})= & \frac{1}{2} \sum_{i} \frac{v_{i}^{2}}{\sigma_{i}^{2}}-\sum_{i j} W_{i j}^{(1)} h_{j}^{(1)} \frac{v_{i}}{\sigma_{i}} \\
& -\sum_{j l} W_{j l}^{(2)} h_{j}^{(1)} h_{l}^{(2)}-\sum_{l k} W_{l k}^{(3)} h_{l}^{(2)} \hat{h}_{k}^{(3)},
\end{aligned}
$$

where $\mathbf{h}=\left\{\mathbf{h}^{(1)}, \mathbf{h}^{(2)}, \mathbf{h}^{(3)}\right\}$ represent the set of hidden units, and $\boldsymbol{\psi}=\left\{\mathbf{W}^{(1)}, \mathbf{W}^{(2)}, \mathbf{W}^{(3)}, \boldsymbol{\sigma}^{2}\right\}$ are the model parameters, and $\sigma_{i}^{2}$ is the variance of input $i$. The marginal distribution over the visible vector $\mathrm{v}$ takes form

$$
P(\mathbf{v} ; \boldsymbol{\psi})=\sum_{\mathbf{h}} \frac{\exp (-E(\mathbf{v}, \mathbf{h} ; \boldsymbol{\psi}))}{\int_{\mathbf{v}^{\prime}} \sum_{\mathbf{h}} \exp (-E(\mathbf{v}, \mathbf{h} ; \boldsymbol{\psi})) d \mathbf{v}^{\prime}} .
$$

From (10), it is straightforward to derive the following conditional distributions:

$$
\begin{aligned}
& p\left(v_{i}=x \mid \mathbf{h}^{(\mathbf{1})}\right)=\frac{1}{\sqrt{2 \pi} \sigma_{i}} \exp \left(-\frac{\left(x-\sigma_{i} \sum_{j} h_{j}^{(1)} W_{i j}^{(1)}\right)^{2}}{2 \sigma_{i}^{2}}\right), \\
& p\left(h_{j}^{(1)}=1 \mid \mathbf{v}\right)=g\left(\sum_{i} W_{i j}^{(1)} \frac{v_{i}}{\sigma_{i}}\right)
\end{aligned}
$$

where $g(x)=1 /(1+\exp (-x))$ is the logistic function. Conditional distributions over $\mathbf{h}^{(2)}$ and $\mathbf{h}^{(3)}$ remain the same as in the standard DBM model (see (2)).

Observe that conditioned on the states of the hidden units (12), each visible unit is modeled by a Gaussian distribution whose mean is shifted by the weighted combination of the hidden unit activations. The derivative of the log-likelihood with respect to $\mathbf{W}^{(1)}$ takes form

$$
\frac{\partial \log P(\mathbf{v} ; \boldsymbol{\psi})}{\partial W_{i j}^{(1)}}=\mathrm{E}_{P_{\text {data }}}\left[\frac{1}{\sigma_{i}} v_{i} h_{j}^{(1)}\right]-\mathrm{E}_{P_{\text {Model }}}\left[\frac{1}{\sigma_{i}} v_{i} h_{j}^{(1)}\right] .
$$

The derivatives with respect to parameters $\mathbf{W}^{(2)}$ and $\mathbf{W}^{(3)}$ remain the same as in (3).

As described in the previous section, learning of the model parameters, including the variances $\boldsymbol{\sigma}^{2}$, can be carried out using variational learning together with stochastic approximation procedure. In practice, however, instead of learning $\boldsymbol{\sigma}^{2}$, one would typically use a fixed, predetermined value for $\boldsymbol{\sigma}^{2}$ [13], [24].

\subsection{Multinomial DBMs}

To allow DBMs to express more information and introduce more structured hierarchical priors, we will use a conditional multinomial distribution to model activities of the top-level units $\mathbf{h}^{(3)}$. Specifically, we will use $M$ softmax units, each with "1-of- $K$ " encoding, so that each unit contains a set of $K$ weights. We represent the $k$ th discrete value of hidden unit by a vector containing 1 at the $k$ th location and zeros elsewhere. The conditional probability of a softmax top-level unit is 


\section{Deep Boltzmann Machine}

$M$ replicated softmax units with tied weights

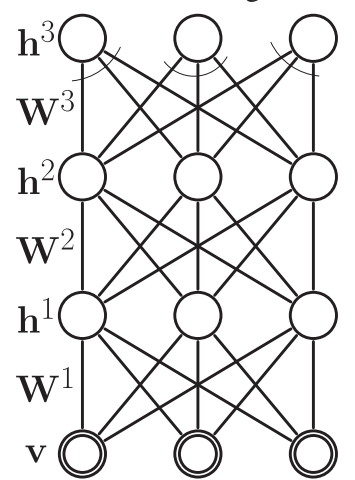

Multinomial unit sampled $M$ times

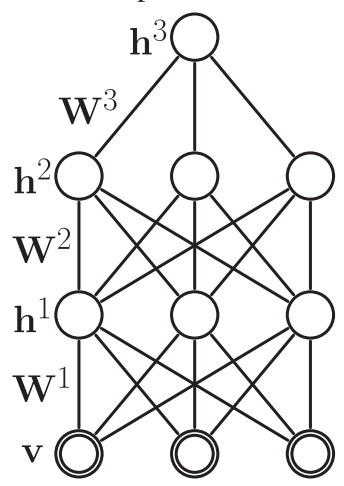

Fig. 1. Left: Multinomial DBM model: The top layer represents M softmax hidden units $\mathbf{h}^{(3)}$ which share the same set of weights. Right: A different interpretation: $M$ softmax units are replaced by a single multinomial unit which is sampled M times.

$$
P\left(h_{k}^{(3)} \mid \mathbf{h}^{(2)}\right)=\frac{\exp \left(\sum_{l} W_{l k}^{(3)} h_{l}^{(2)}\right)}{\sum_{s=1}^{K} \exp \left(\sum_{l} W_{l s}^{(3)} h_{l}^{(2)}\right)} .
$$

In our formulation, all $M$ separate softmax units will share the same set of weights, connecting them to binary hidden units at the lower level (see Fig. 1). The energy of the state $\{\mathbf{v}, \mathbf{h}\}$ is then defined as follows:

$$
\begin{aligned}
E(\mathbf{v}, \mathbf{h} ; \boldsymbol{\psi})= & -\sum_{i j} W_{i j}^{(1)} v_{i} h_{j}^{(1)}-\sum_{j l} W_{j l}^{(2)} h_{j}^{(1)} h_{l}^{(2)} \\
& -\sum_{l k} W_{l k}^{(3)} h_{l}^{(2)} \hat{h}_{k}^{(3)},
\end{aligned}
$$

where $\mathbf{h}^{(1)} \in\{0,1\}^{F_{1}}$ and $\mathbf{h}^{(2)} \in\{0,1\}^{F_{2}}$ represent stochastic binary units. The top layer is represented by the M softmax units $\mathbf{h}^{(3, m)}, m=1, \ldots, M$, with $\hat{h}_{k}^{(3)}=\sum_{m=1}^{M} h_{k}^{(3, m)}$ denoting the count for the $k$ th discrete value of a hidden unit.

A key observation is that $M$ separate copies of softmax units that all share the same set of weights can be viewed as a single multinomial unit that is sampled $\mathrm{M}$ times from the conditional distribution of (13). This gives us a familiar "bag-of-words" representation [30], [36]. A pleasing property of using softmax units is that the mathematics underlying the learning algorithm for binary-binary DBMs remains the same.

\section{Compound HDP-DBM MOdeL}

After a DBM model has been learned, we have an undirected model that defines the joint distribution $P\left(\mathbf{v}, \mathbf{h}^{(1)}, \mathbf{h}^{(2)}, \mathbf{h}^{(3)}\right)$. One way to express what has been learned is the conditional model $P\left(\mathbf{v}, \mathbf{h}^{(1)}, \mathbf{h}^{(2)} \mid \mathbf{h}^{(3)}\right)$ and a complicated prior term $P\left(\mathbf{h}^{(3)}\right)$, defined by the DBM model. We can therefore rewrite the variational bound as

$$
\begin{aligned}
\log P(\mathbf{v}) \geq & \sum_{\mathbf{h}^{(1)}, \mathbf{h}^{(2)}, \mathbf{h}^{(3)}} Q(\mathbf{h} \mid \mathbf{v} ; \boldsymbol{\mu}) \log P\left(\mathbf{v}, \mathbf{h}^{(1)}, \mathbf{h}^{(2)} \mid \mathbf{h}^{(3)}\right) \\
& +\mathcal{H}(Q)+\sum_{\mathbf{h}^{(3)}} Q\left(\mathbf{h}^{(3)} \mid \mathbf{v} ; \boldsymbol{\mu}\right) \log P\left(\mathbf{h}^{(3)}\right) .
\end{aligned}
$$

This particular decomposition lies at the core of the greedy recursive pretraining algorithm: We keep the learned condi-

tional model $P\left(\mathbf{v}, \mathbf{h}^{(1)}, \mathbf{h}^{(2)} \mid \mathbf{h}^{(3)}\right)$, but maximize the variational lower bound of (14) with respect to the last term [12]. This maximization amounts to replacing $P\left(\mathbf{h}^{(3)}\right)$ by a prior that is closer to the average, over all the data vectors, of the approximate conditional posterior $Q\left(\mathbf{h}^{(3)} \mid \mathbf{v}\right)$.

Instead of adding an additional undirected layer (e.g., an RBM) to model $P\left(\mathbf{h}^{(3)}\right)$ we can place an HDP prior over $\mathbf{h}^{(3)}$ that will allow us to learn category hierarchies and, more importantly, useful representations of classes that contain few training examples.

The part we keep, $P\left(\mathbf{v}, \mathbf{h}^{(1)}, \mathbf{h}^{(2)} \mid \mathbf{h}^{(3)}\right)$, represents a conditional DBM model: ${ }^{5}$

$$
\begin{aligned}
P\left(\mathbf{v}, \mathbf{h}^{(1)}, \mathbf{h}^{(2)} \mid \mathbf{h}^{(3)}\right)= & \frac{1}{\mathcal{Z}\left(\boldsymbol{\psi}, \mathbf{h}^{(3)}\right)} \exp \left(\sum_{i j} W_{i j}^{(1)} v_{i} h_{j}^{(1)}\right. \\
& \left.+\sum_{j l} W_{j l}^{(2)} h_{j}^{(1)} h_{l}^{(2)}+\sum_{l k} W_{l k}^{(3)} h_{l}^{(2)} h_{k}^{(3)}\right),
\end{aligned}
$$

which can be viewed as a two-layer DBM but with bias terms given by the states of $\mathbf{h}^{(3)}$.

\subsection{A Hierarchical Bayesian Prior}

In a typical hierarchical topic model, we observe a set of $N$ documents, each of which is modeled as a mixture over topics, that are shared among documents. Let there be $K$ words in the vocabulary. A topic $t$ is a discrete distribution over $K$ words with probability vector $\boldsymbol{\phi}_{t}$. Each document $n$ has its own distribution over topics given by probabilities $\boldsymbol{\theta}_{n}$.

In our compound HDP-DBM model, we will use a hierarchical topic model as a prior over the activities of the DBM's top-level features. Specifically, the term "document" will refer to the top-level multinomial unit $\mathbf{h}^{(3)}$, and $M$ "words" in the document will represent the $M$ samples, or active DBM's top-level features, generated by this multinomial unit. Words in each document are drawn by choosing a topic $t$ with probability $\theta_{n t}$, and then choosing a word $w$ with probability $\phi_{t w}$. We will often refer to topics as our learned higher level features, each of which defines a topic specific distribution over DBM's $\mathbf{h}^{(3)}$ features. Let $h_{i n}^{(3)}$ be the $i$ th word in document $n$, and $x_{i n}$ be its topic. We can specify the following prior over $\mathbf{h}^{(3)}$ :

$$
\begin{aligned}
\boldsymbol{\theta}_{n} \mid \boldsymbol{\pi} & \sim \operatorname{Dir}(\alpha \boldsymbol{\pi}), \text { for each document } n=1, \ldots, N, \\
\boldsymbol{\phi}_{t} \mid \boldsymbol{\tau} & \sim \operatorname{Dir}(\beta \boldsymbol{\tau}), \text { for each topic } \mathrm{t}=1, \ldots, T, \\
x_{i n} \mid \boldsymbol{\theta}_{n} & \sim \operatorname{Mult}\left(1, \boldsymbol{\theta}_{n}\right), \text { for each word } \mathrm{i}=1, \ldots, M, \\
h_{i n}^{(3)} \mid x_{i n}, \boldsymbol{\phi}_{x_{i n}} & \sim \operatorname{Mult}\left(1, \boldsymbol{\phi}_{x_{i n}}\right),
\end{aligned}
$$

where $\pi$ is the global distribution over topics, $\boldsymbol{\tau}$ is the global distribution over $K$ words, and $\alpha$ and $\beta$ are concentration parameters.

Let us further assume that our model is presented with a fixed two-level category hierarchy. In particular, suppose that $N$ documents, or objects, are partitioned into $C$ basic level categories (e.g., cow, sheep, car). We represent such a partition by a vector $\mathbf{z}^{b}$ of length $N$, each entry of which is $z_{n}^{b} \in\{1 \ldots C\}$. We also assume that our $C$ basic-level

5. Our experiments reveal that using DBNs instead of DBMs decreased model performance. 


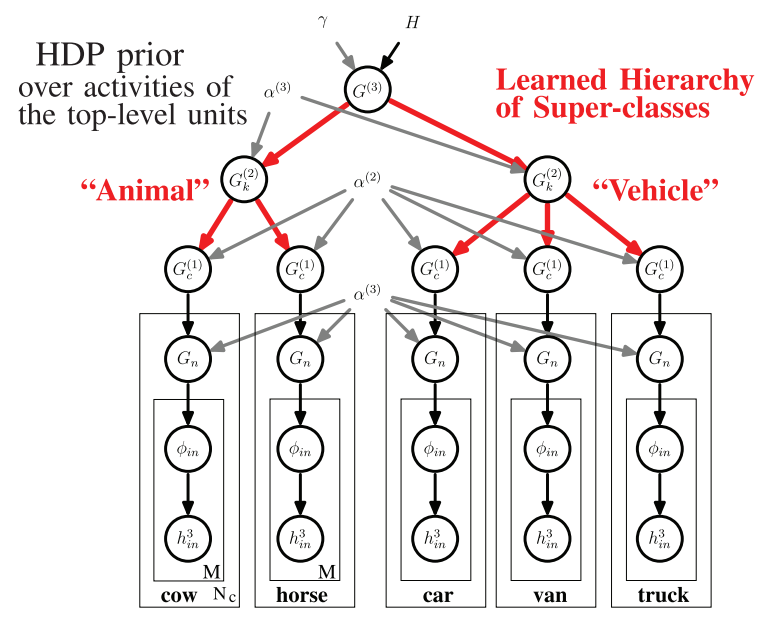

Fig. 2. HDP prior over the states of the DBM's top-level features $\mathbf{h}^{(3)}$.

categories are partitioned into $S$ supercategories (e.g., animal, vehicle), represented by a vector $\mathbf{z}^{s}$ of length $C$, with $z_{c}^{s} \in\{1 \ldots S\}$. These partitions define a fixed two-level tree hierarchy (see Fig. 2). We will relax this assumption later by placing a nonparametric prior over the category assignments.

The hierarchical topic model can be readily extended to modeling the above hierarchy. For each document $n$ that belongs to the basic category $c$, we place a common Dirichlet prior over $\boldsymbol{\theta}_{n}$ with parameters $\boldsymbol{\pi}_{c}^{(1)}$. The Dirichlet parameters $\boldsymbol{\pi}^{(1)}$ are themselves drawn from a Dirichlet prior with level-2 parameters $\boldsymbol{\pi}^{(2)}$, common to all basic-level categories that belong to the same supercategory, and so on. Specifically, we define the following hierarchical prior over $\mathbf{h}^{(3)}$ :

$\boldsymbol{\pi}_{s}^{(2)} \mid \boldsymbol{\pi}_{g}^{(3)} \sim \operatorname{Dir}\left(\alpha^{(3)} \pi_{g}^{(3)}\right)$, for each superclass $s=1, \ldots, S$, $\boldsymbol{\pi}_{c}^{(1)} \mid \boldsymbol{\pi}_{z_{c}^{s}}^{(2)} \sim \operatorname{Dir}\left(\alpha^{(2)} \boldsymbol{\pi}_{z_{c}^{s}}^{(2)}\right)$, for each basic-classc $=1, \ldots, C$, $\boldsymbol{\theta}_{n} \mid \boldsymbol{\pi}_{z_{n}^{b}}^{(1)} \sim \operatorname{Dir}\left(\alpha^{(1)} \boldsymbol{\pi}_{z_{n}^{b}}^{(1)}\right)$, for each document $n=1, \ldots, N$, $x_{i n} \mid \boldsymbol{\theta}_{n} \sim \operatorname{Mult}\left(1, \boldsymbol{\theta}_{n}\right), \quad$ for each word $i=1, \ldots, M$, $\boldsymbol{\phi}_{t} \mid \beta, \boldsymbol{\tau} \sim \operatorname{Dir}(\beta \boldsymbol{\tau})$, $h_{i n}^{(3)} \mid x_{i n}, \boldsymbol{\phi}_{x_{i n}} \sim \operatorname{Mult}\left(1, \boldsymbol{\phi}_{x_{i n}}\right)$,

where $\boldsymbol{\pi}_{g}^{(3)}$ is the global distribution over topics, $\boldsymbol{\pi}_{s}^{(2)}$ is the supercategory specific, and $\boldsymbol{\pi}_{c}^{(1)}$ is the class specific distribution over topics, or higher level features. These high-level features in turn define topic-specific distribution over $\mathbf{h}^{(3)}$ features, or "words" in our DBM model. Finally, $\alpha^{(1)}, \alpha^{(2)}$, and $\alpha^{(3)}$ represent concentration parameters describing how close $\pi \mathrm{s}$ are to their respective prior means within the hierarchy.

For a fixed number of topics $T$, the above model represents a hierarchical extension of the latent Dirichlet allocation (LDA) model [4]. However, we typically do not know the number of topics a-priori. It is therefore natural to consider a nonparametric extension based on the HDP model [38], which allows for a countably infinite number of topics. In the standard HDP notation, we have the following:

$$
\begin{aligned}
G_{g}^{(3)} \mid \beta, \gamma, \tau & \sim \operatorname{DP}(\gamma, \operatorname{Dir}(\beta \boldsymbol{\tau})), \\
G_{s}^{(2)} \mid \alpha^{(3)}, G^{(3)} & \sim \operatorname{DP}\left(\alpha^{(3)}, G_{g}^{(3)}\right), \\
G_{c}^{(1)} \mid \alpha^{(2)}, G^{(2)} & \sim \operatorname{DP}\left(\alpha^{(2)}, G_{z_{c}^{s}}^{(2)}\right), \\
G_{n} \mid \alpha^{(1)}, G^{(1)} & \sim \operatorname{DP}\left(\alpha^{(1)}, G_{z_{n}^{b}}^{(1)}\right), \\
\boldsymbol{\phi}_{i n}^{*} \mid G_{n} & \sim G_{n}, \\
h_{i n}^{3} \mid \boldsymbol{\phi}_{i n}^{*} & \sim \operatorname{Mult}\left(1, \boldsymbol{\phi}_{i n}^{*}\right),
\end{aligned}
$$

where $\operatorname{Dir}(\beta \boldsymbol{\tau})$ is the base-distribution, and each $\boldsymbol{\phi}^{*}$ is a factor associated with a single observation $h_{i n}^{(3)}$. Making use of topic index variables $x_{i n}$, we denote $\boldsymbol{\phi}_{i n}^{*}=\boldsymbol{\phi}_{x_{i n}}$ (see (16)). Using a stick-breaking representation, we can write

$$
\begin{aligned}
G_{g}^{(3)}(\phi) & =\sum_{t=1}^{\infty} \pi_{g t}^{(3)} \delta_{\phi_{t}}, G_{s}^{(2)}(\phi) \sum_{t=1}^{\infty} \pi_{s t}^{(2)} \delta_{\phi_{t}}, \\
G_{c}^{(1)}(\phi) & =\sum_{t=1}^{\infty} \pi_{c t}^{(1)} \delta_{\phi_{t}}, G_{n}(\phi)=\sum_{t=1}^{\infty} \theta_{n t} \delta_{\phi_{t}},
\end{aligned}
$$

which represent sums of point masses. We also place Gamma priors over concentration parameters as in [38].

The overall generative model is shown in Fig. 2. To generate a sample, we first draw $M$ words, or activations of the top-level features, from the HDP prior over $\mathbf{h}^{(3)}$ given by (17). Conditioned on $\mathbf{h}^{(3)}$, we sample the states of $\mathbf{v}$ from the conditional DBM model given by (15).

\subsection{Modeling the Number of Supercategories}

So far we have assumed that our model is presented with a two-level partition $\mathbf{z}=\left\{\mathbf{z}^{s}, \mathbf{z}^{b}\right\}$ that defines a fixed two-level tree hierarchy. We note that this model corresponds to a standard HDP model that assumes a fixed hierarchy for sharing parameters. If, however, we are not given any level-1 or level-2 category labels, we need to infer the distribution over the possible category structures. We place a nonparametric two-level nested Chinese restaurant prior (CRP) [5] over $\mathbf{z}$, which defines a prior over tree structures and is flexible enough to learn arbitrary hierarchies. The main building block of the nested CRP is the Chinese restaurant process, a distribution on partition of integers. Imagine a process by which customers enter a restaurant with an unbounded number of tables, where the $n$th customer occupies a table $k$ drawn from

$$
P\left(z_{n}=k \mid z_{1} \ldots z_{n-1}\right)= \begin{cases}\frac{n^{k}}{n-1+\eta}, & n^{k}>0, \\ \frac{\eta}{n-1+\eta}, & k \text { is new },\end{cases}
$$

where $n^{k}$ is the number of previous customers at table $k$ and $\eta$ is the concentration parameter.

The nested CRP, $\operatorname{nCRP}(\eta)$, extends CRP to nested sequence of partitions, one for each level of the tree. In this case each observation $n$ is first assigned to the supercategory $z_{n}^{s}$ using (19). Its assignment to the basiclevel category $z_{n}^{b}$, which is placed under a supercategory $z_{n}^{s}$, is again recursively drawn from (19). We also place a Gamma prior $\Gamma(1,1)$ over $\eta$. The proposed model allows for both: a nonparametric prior over potentially unbounded number of global topics, or higher level features, as well as a nonparametric prior that allows learning an arbitrary tree taxonomy. 
Unlike in many conventional HB models, here we infer both the model parameters as well as the hierarchy for sharing those parameters. As we show in the experimental results section, both sharing higher level features and forming coherent hierarchies play a crucial role in the ability of the model to generalize well from one or few examples of a novel category. Our model can be readily used in unsupervised or semi-supervised modes, with varying amounts of label information at different levels of the hierarchy.

\section{INFERENCE}

Inferences about model parameters at all levels of hierarchy can be performed by MCMC. When the tree structure $\mathbf{z}$ of the model is not given, the inference process will alternate between fixing $\mathbf{z}$ while sampling the space of model parameters, and vice versa.

Sampling HDP parameters. Given the category assignment vector $\mathbf{z}$ and the states of the top-level DBM features $\mathbf{h}^{(3)}$, we use the posterior representation sampler of [37]. In particular, the HDP sampler maintains the stick-breaking weights $\{\boldsymbol{\theta}\}_{n=1}^{N},\left\{\boldsymbol{\pi}_{c}^{(1)}, \boldsymbol{\pi}_{s}^{(2)}, \boldsymbol{\pi}_{g}^{(3)}\right\}$ and topic indicator variables $\mathbf{x}$ (parameters $\phi$ can be integrated out). The sampler alternates between: 1) sampling cluster indices $x_{i n}$ using Gibbs updates in the Chinese restaurant franchise (CRF) representation of the HDP; 2) sampling the weights at all three levels conditioned on $\mathrm{x}$ using the usual posterior of a DP.

Conditioned on the draw of the superclass DP $G_{s}^{(2)}$ and the state of the CRF, the posteriors over $G_{c}^{(1)}$ become independent. We can easily speed up inference by sampling from these conditionals in parallel. The speedup could be substantial, particularly as the number of the basic-level categories becomes large.

Sampling category assignments z. Given the current instantiation of the stick-breaking weights, for each input $n$ we have

$$
\begin{aligned}
& \left(\theta_{1, n}, \ldots, \theta_{T, n}, \theta_{\text {new }, n}\right) \sim \\
& \operatorname{Dir}\left(\alpha^{(1)} \pi_{\mathbf{z}_{n}, 1}^{(1)}, \ldots, \alpha^{(1)} \pi_{\mathbf{z}_{n}, T}^{(1)}, \alpha^{(1)} \pi_{\mathbf{z}_{n}, \text { new }}^{(1)}\right) .
\end{aligned}
$$

Combining the above likelihood term with the CRP prior (19), the posterior over the category assignment can be calculated as follows:

$$
p\left(\mathbf{z}_{n} \mid \boldsymbol{\theta}_{n}, \mathbf{z}_{-n}, \boldsymbol{\pi}^{(1)}\right) \propto p\left(\boldsymbol{\theta}_{n} \mid \boldsymbol{\pi}^{(1)}, \mathbf{z}_{n}\right) p\left(\mathbf{z}_{n} \mid \mathbf{z}_{-n}\right),
$$

where $\mathbf{z}_{-n}$ denotes variables $\mathbf{z}$ for all observations other than $n$. When computing the probability of placing $\boldsymbol{\theta}_{n}$ under a newly created category, its parameters are sampled from the prior.

Sampling DBM's hidden units. Given the states of the DBM's top-level multinomial unit $\mathbf{h}_{n}^{(3)}$, conditional samples from $P\left(\mathbf{h}_{n}^{(1)}, \mathbf{h}_{n}^{(2)} \mid \mathbf{h}_{n}^{(3)}, \mathbf{v}_{n}\right)$ can be obtained by running a Gibbs sampler that alternates between sampling the states of $\mathbf{h}_{n}^{(1)}$ independently given $\mathbf{h}_{n}^{(2)}$, and vice versa. Conditioned on topic assignments $x_{i n}$ and $\mathbf{h}_{n}^{(2)}$, the states of the multinomial unit $\mathbf{h}_{n}^{(3)}$ for each input $n$ are sampled using Gibbs conditionals:

$$
P\left(\mathbf{h}_{i n}^{(3)} \mid \mathbf{h}_{n}^{(2)}, \mathbf{h}_{-i n}^{(3)}, \mathbf{x}_{n}\right) \propto P\left(\mathbf{h}_{n}^{(2)} \mid \mathbf{h}_{n}^{(3)}\right) P\left(\mathbf{h}_{i n}^{(3)} \mid \mathbf{x}_{i n}\right),
$$

where the first term is given by the product of logistic functions (see (15)):

$$
\begin{gathered}
P\left(\mathbf{h}_{n}^{(2)} \mid \mathbf{h}_{n}^{(3)}\right)=\prod_{l} P\left(h_{l n}^{(2)} \mid \mathbf{h}_{n}^{(3)}\right), \text { with } \\
P\left(h_{l}^{(2)}=1 \mid \mathbf{h}^{(3)}\right)=\frac{1}{1+\exp \left(-\sum_{k} W_{l k}^{(3)} h_{k}^{(3)}\right)},
\end{gathered}
$$

and the second term $P\left(\mathbf{h}_{i n}^{(3)}\right)$ is given by the multinomial: $\operatorname{Mult}\left(1, \boldsymbol{\phi}_{x_{i n}}\right)$ (see (17)). In our conjugate setting, parameters $\phi$ can be further integrated out.

Fine-tuning DBM. Finally, conditioned on the states of $\mathbf{h}^{(3)}$, we can further fine-tune low-level DBM parameters $\boldsymbol{\psi}=\left\{\mathbf{W}^{(1)}, \mathbf{W}^{(2)}, \mathbf{W}^{(3)}\right\}$ by applying approximate maximum likelihood learning (see Section 2) to the conditional DBM model of (15). For the stochastic approximation algorithm, since the partition function depends on the states of $\mathbf{h}^{(3)}$, we maintain one "persistent" Markov chain per data point (for details see [29], [39]). As we show in our experimental results section, fine-tuning low-level DBM features can significantly improve model performance.

\subsection{Making Predictions}

Given a test input $\mathbf{v}_{t}$, we can quickly infer the approximate posterior over $\mathbf{h}_{t}^{(3)}$ using the mean-field of (6), followed by running the full Gibbs sampler to get approximate samples from the posterior over the category assignments. In practice, for faster inference, we fix learned topics $\phi_{t}$ and approximate the marginal likelihood that $\mathbf{h}_{t}^{(3)}$ belongs to category $\mathbf{z}_{t}$ by assuming that document specific DP can be well approximated by the class-specific ${ }^{6}$ DP $G_{t} \approx G_{\mathbf{z}_{t}}^{(1)}$ (see Fig. 2). Hence, instead of integrating out document specific DP $G_{t}$, we approximate

$$
\begin{aligned}
P\left(\mathbf{h}_{t}^{(3)} \mid \mathbf{z}_{t}, G^{(1)}, \boldsymbol{\phi}\right) & =\int_{G_{t}} P\left(\mathbf{h}_{t}^{(3)} \mid \boldsymbol{\phi}, G_{t}\right) P\left(G_{t} \mid G_{\mathbf{z}_{t}}^{(1)}\right) d G_{t} \\
& \approx P\left(\mathbf{h}_{t}^{(3)} \mid \boldsymbol{\phi}, G_{\mathbf{z}_{t}}^{(1)}\right),
\end{aligned}
$$

which can be computed analytically by integrating out topic assignments $x_{i n}$ (17). Combining this likelihood term with nCRP prior $P\left(\mathbf{z}_{t} \mid \mathbf{z}_{-t}\right)$ of (19) allows us to efficiently infer approximate posterior over category assignments. In all of our experimental results, computing this approximate posterior takes a fraction of a second, which is crucial for applications, such as object recognition or information retrieval.

\section{EXPERIMENTS}

We present experimental results on the CIFAR-100 [17], handwritten character [18], and human motion capture recognition datasets. For all datasets, we first pretrain a DBM model in unsupervised fashion on raw sensory input (e.g., pixels, or three-dimensional joint angles), followed by fitting an HDP prior which is run for 200 Gibbs sweeps. We further run 200 additional Gibbs steps to fine-tune parameters of the entire compound HDP-DBM model. This was sufficient to obtain good performance. Across all datasets, we also assume that the basic-level category

6. We note that $G_{\mathbf{z}_{t}}^{(1)}=\mathrm{E}\left[G_{t} \mid G_{\mathbf{z}_{t}}^{(1)}\right]$. 

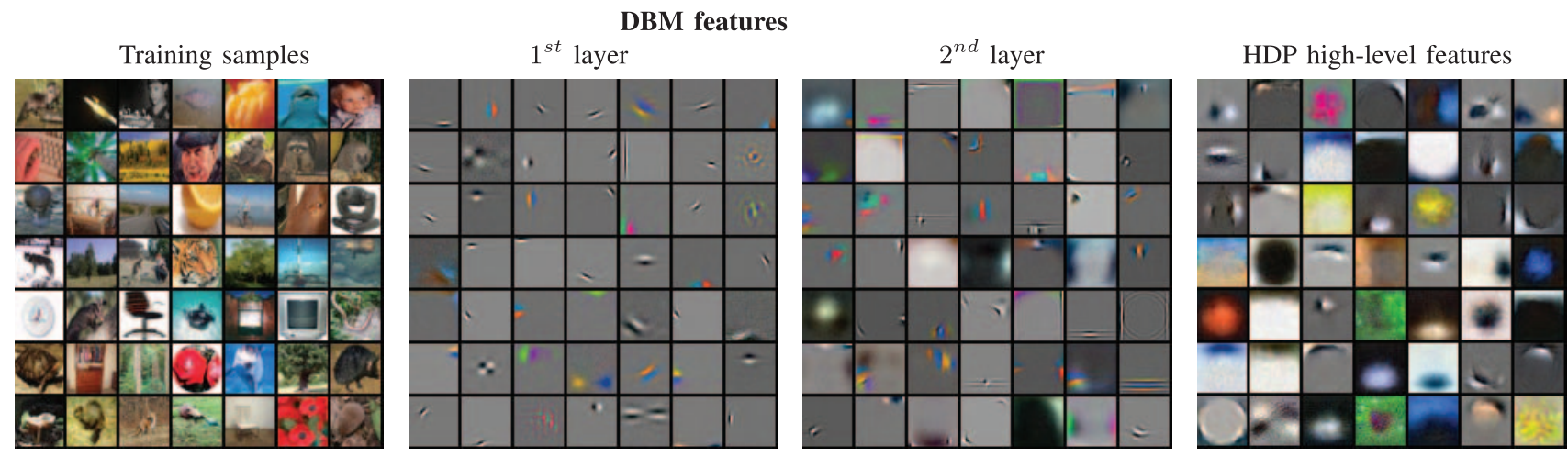

Fig. 3. A random subset of the training images along with the first and second layer DBM features and higher level class-sensitive HDP features/ topics. To visualize higher level features, we first sample $M$ words from a fixed topic $\phi_{t}$, followed by sampling RGB pixel values from the conditional DBM model.

labels are given, but no supercategory labels are available. We must infer how to cluster basic categories into supercategories at the same time as we infer parameter values at all levels of the hierarchy. The training set includes many examples of familiar categories but only a few examples of a novel class. Our goal is to generalize well on a novel class.

In all experiments, we compare performance of HDPDBM to the following alternative models. The first two models, stand-alone DBMs and DBNs [12], used three layers of hidden variables and were pretrained using a stack of RBMs. To evaluate classification performance of DBNs and DBMs, both models were converted into multilayer neural networks and were discriminatively fine-tuned using backpropagation algorithm (see [29] for details). Our third model, "Flat HDP-DBM," always used a single supercategory. The Flat HDP-DBM approach, similar in spirit to the one-shot learning model of [11], could potentially identify a set of useful high-level features common to all categories. Our fourth model used a version of SVM that implements cost-sensitive learning. ${ }^{7}$ The basic idea is to assign a larger penalty value for misclassifying examples that arise from the underrepresented class. In our setting, this model performs slightly better compared to a standard SVM classifier. Our last model used a simple $k$ nearest neighbors (k-NN) classifier. Finally, using HDPs on top of raw sensory input (i.e., pixels, or even imagespecific GIST features) performs far worse compared to our HDP-DBM model.

\subsection{CIFAR-100 Data Set}

The CIFAR-100 image dataset [17] contains 50,000 training and 10,000 test images of 100 object categories (100 per class), with $32 \times 32 \times 3$ RGB pixels. Extreme variability in scale, viewpoint, illumination, and cluttered background makes the object recognition task for this dataset quite difficult. Similarly to [17], to learn good generic low-level features, we first train a two-layer DBM in completely unsupervised fashion using 4 million tiny images ${ }^{8}$ [40]. We use a conditional Gaussian distribution to model observed pixel values [13], [17]. The first DBM layer

7. We used LIBSVM software package of [7].

8. The dataset contains random images of natural scenes downloaded from the web. contained 10,000 binary hidden units, and the second layer contained $\mathrm{M}=1,000$ softmax units. ${ }^{9}$ We then fit an HDP prior over $\mathbf{h}^{(2)}$ to the 100 object classes. We also experimented with a three-layer DBM model, as well as various softmax parameters: $M=500$ and $M=2,000$. The difference in performance was not significant.

Fig. 3 displays a random subset of the training data, first and second layer DBM features, as well as higher level class-sensitive features, or topics, learned by the HDP model. Second layer features were visualized as a weighted linear combination of the first layer features as in [21]. To visualize a particular higher level feature, we first sample $M$ words from a fixed topic $\phi_{t}$, followed by sampling RGB pixel values from the conditional DBM model. While DBM features capture mostly low-level structure, including edges and corners, the HDP features tend to capture higher level structure, including contours, shapes, color components, and surface boundaries in the images. More importantly, features at all levels of the hierarchy evolve without incorporating any image-specific priors. Fig. 4 shows a typical partition over 100 classes that our model discovers with many supercategories containing semantically similar classes.

Table 1 quantifies performance using the area under the ROC curve (AUROC) for classifying 10,000 test images as belonging to the novel versus all of the other 99 classes. We report $2 *$ AUROC-1, so zero corresponds to the classifier that makes random predictions. The results are averaged over 100 classes using "leave-one-out" test format. Based on a single example, the HDP-DBM model achieves an AUROC of 0.36, significantly outperforming DBMs, DBNs, SVMs, and 1-NN using standard image-specific GIST features ${ }^{10}$ that achieve an AUROC of 0.26, 0.25, 0.20, and 0.27 , respectively. Table 1 also shows that fine-tuning parameters of all layers jointly as well as learning supercategory hierarchy significantly improves model performance. As the number of training examples increases, the HDP-DBM model still outperforms alternative methods.

9. The generative training of the DBM model using 4 million images takes about a week on the Intel Xeon $3.00 \mathrm{GHz}$. Fitting an HDP prior to the DBMs top-level features on the CIFAR dataset takes about 12 hours. However, at test time, using variational inference and approximation of (24), it takes a fraction of a second to classify a test example into its corresponding category.

10. Gist descriptors have previously been used for this dataset [41]. 
1. bed, chair, clock, couch, dinosaur, lawn mower, table, telephone, television, wardrobe

2. bus, house, pickup truck, streetcar, tank, tractor, train

3. crocodile, kangaroo, lizard, snake, spider, squirrel

4. hamster, mouse, rabbit, raccoon, possum, bear

5. apple, orange, pear, sunflower, sweet pepper

6. baby, boy, girl, man, woman

7. dolphin, ray, shark, turtle, whale

8. otter, porcupine, shrew, skunk

9. beaver, camel, cattle, chimpanzee, elephant

10. fox, leopard, lion, tiger, wolf

11. maple tree, oak tree, pine tree, willow tree

12 flatfish, seal, trout, worm

13 butterfly, caterpillar, snail

14 bee, crab, lobster

15 bridge, castle, road, skyscraper

16 bicycle, keyboard, motorcycle, orchid, palm tree

17 bottle, bowl, can, cup, lamp

18 cloud, plate, rocket 19. mountain, plain, sea

20 poppy, rose, tulip 21. aquarium fish, mushroom

22 beetle, cockroach 23. forest

Fig. 4. A typical partition of the 100 basic-level categories. Many of the discovered supercategories contain semantically coherent classes.

With 50 training examples, however, all models perform about the same. This is to be expected, as with more training examples, the effect of the hierarchical prior decreases.

We next illustrate the ability of the HDP-DBM to generalize from a single training example of a "pear" class. We trained the model on 99 classes containing 500 training images each, but only one training example of a "pear" class. Fig. 5 shows the kind of transfer our model is performing, where we display training examples along with eight most probable topics $\phi_{t}$, ordered by hand. The model discovers that pears are like apples and oranges, and not like other classes of images, such as dolphins, that reside in very different parts of the hierarchy. Hence the novel category can inherit the prior distribution over similar highlevel shape and color features, allowing the HDP-DBM to generalize considerably better to new instances of the "pear" class.

We next examined the generative performance of the HDP-DBM model. Fig. 6 shows samples generated by the HDP-DBM model for four classes: "Apple," "Willow Tree," "Elephant," and "Castle." Despite extreme variability in
Shared HDP high-level features

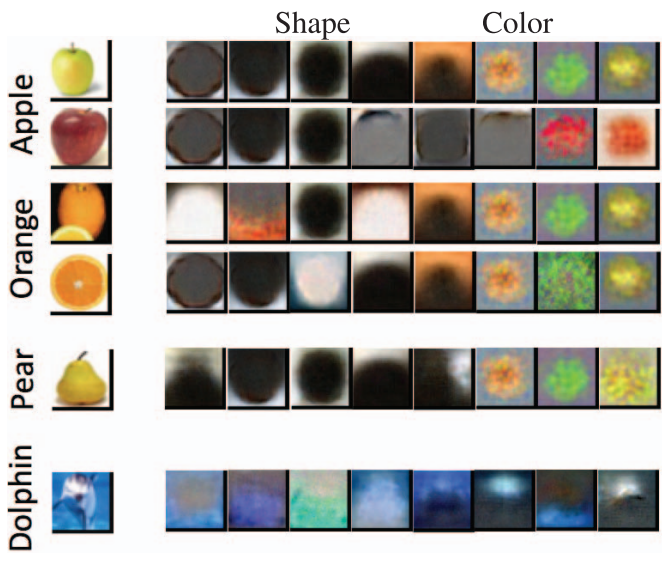

Fig. 5. Learning to learn: Training examples along with the eight most probable topics $\phi_{t}$, ordered by hand.

scale, viewpoint, and cluttered background, the model is able to capture the overall structure of each class. Fig. 7 shows conditional samples when learning with only three training examples of a novel class. For example, based on only three training examples of the "Apple" class, the HDPDBM model is able to generate a rich variety of new apples. Fig. 8 further quantifies performance of HDP-DBM, DBM, and SVM models for all object categories when learning with only three examples. Observe that over 40 classes benefit in various degrees from both: learning a hierarchy as well as learning low and high-level features.

\subsection{Handwritten Characters}

The handwritten characters dataset [18] can be viewed as the "transpose" of the standard MNIST dataset. Instead of containing 60,000 images of 10 digit classes, the dataset contains 30,000 images of 1,500 characters (20 examples each) with $28 \times 28$ pixels. These characters are from 50 alphabets from around the world, including Bengali, Cyrillic, Arabic, Sanskrit, Tagalog (see Fig. 9). We split the dataset into 15,000 training and 15,000 test images (10 examples of each class). Similarly to the CIFAR dataset, we pretrain a two-layer DBM model, with the first layer containing 1,000 hidden units, and the second layer containing $\mathrm{M}=100$ softmax units. The HDP prior over $\mathbf{h}^{(2)}$ was fit to all 1,500 character classes.

TABLE 1

Classification Performance on the Test Set Using $2^{*}$ AUROC -1

\begin{tabular}{|c|c|c|c|c|c|c|c|c|c|c|c|c|c|c|}
\hline \multirow[b]{2}{*}{ Model } & \multicolumn{5}{|c|}{$\begin{array}{c}\text { CIFAR Dataset } \\
\text { Number of examples }\end{array}$} & \multicolumn{4}{|c|}{$\begin{array}{l}\text { Handwritten Characters } \\
\text { Number of examples }\end{array}$} & \multicolumn{5}{|c|}{$\begin{array}{c}\text { Motion Capture } \\
\text { Number of examples }\end{array}$} \\
\hline & 1 & 3 & 5 & 10 & 50 & 1 & 3 & 5 & 10 & 1 & 3 & 5 & 10 & 50 \\
\hline Tuned HDP-DBM & 0.36 & 0.41 & 0.46 & 0.53 & 0.62 & 0.67 & 0.78 & 0.87 & 0.93 & 0.67 & 0.84 & 0.90 & 0.93 & 0.96 \\
\hline HDP-DBM & 0.34 & 0.39 & 0.45 & 0.52 & 0.61 & 0.65 & 0.76 & 0.85 & 0.92 & 0.66 & 0.82 & 0.88 & 0.93 & 0.96 \\
\hline Flat HDP-DBM & 0.27 & 0.37 & 0.42 & 0.50 & 0.61 & 0.58 & 0.73 & 0.82 & 0.89 & 0.63 & 0.79 & 0.86 & 0.91 & 0.96 \\
\hline DBM & 0.26 & 0.36 & 0.41 & 0.48 & 0.61 & 0.57 & 0.72 & 0.81 & 0.89 & 0.61 & 0.79 & 0.85 & 0.91 & 0.95 \\
\hline DBN & 0.25 & 0.33 & 0.37 & 0.45 & 0.60 & 0.51 & 0.72 & 0.81 & 0.89 & 0.61 & 0.79 & 0.84 & 0.92 & 0.96 \\
\hline SVM & 0.20 & 0.29 & 0.32 & 0.39 & 0.61 & 0.43 & 0.68 & 0.78 & 0.87 & 0.55 & 0.78 & 0.85 & 0.91 & 0.96 \\
\hline $1-\mathrm{NN}$ & 0.17 & 0.18 & 0.19 & 0.20 & 0.32 & 0.43 & 0.65 & 0.73 & 0.81 & 0.58 & 0.75 & 0.81 & 0.88 & 0.93 \\
\hline GIST & 0.27 & 0.31 & 0.33 & 0.39 & 0.58 & - & - & - & - & - & - & - & - & \\
\hline
\end{tabular}

The results in bold correspond to ROCs that are statistically indistinguishable from the best (the difference is not statistically significant). 


\section{Generated Samples}
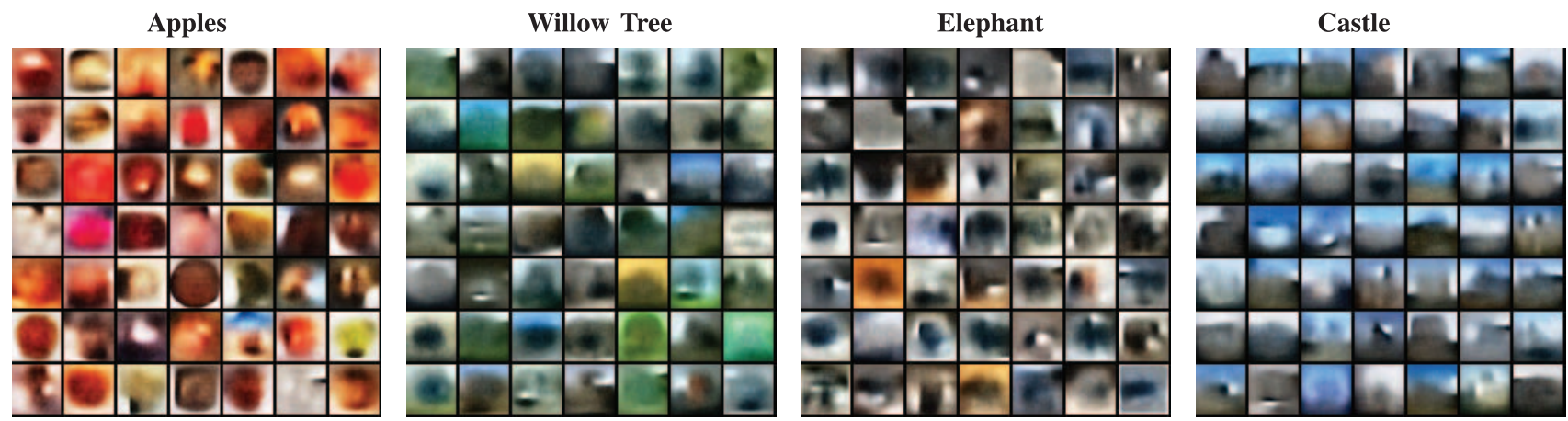

Fig. 6. Class-conditional samples generated from the HDP-DBM model. Observe that the model despite extreme variability, the model is able to capture a coherent structure of each class. See in color for better visualization.

Learning with Three Examples
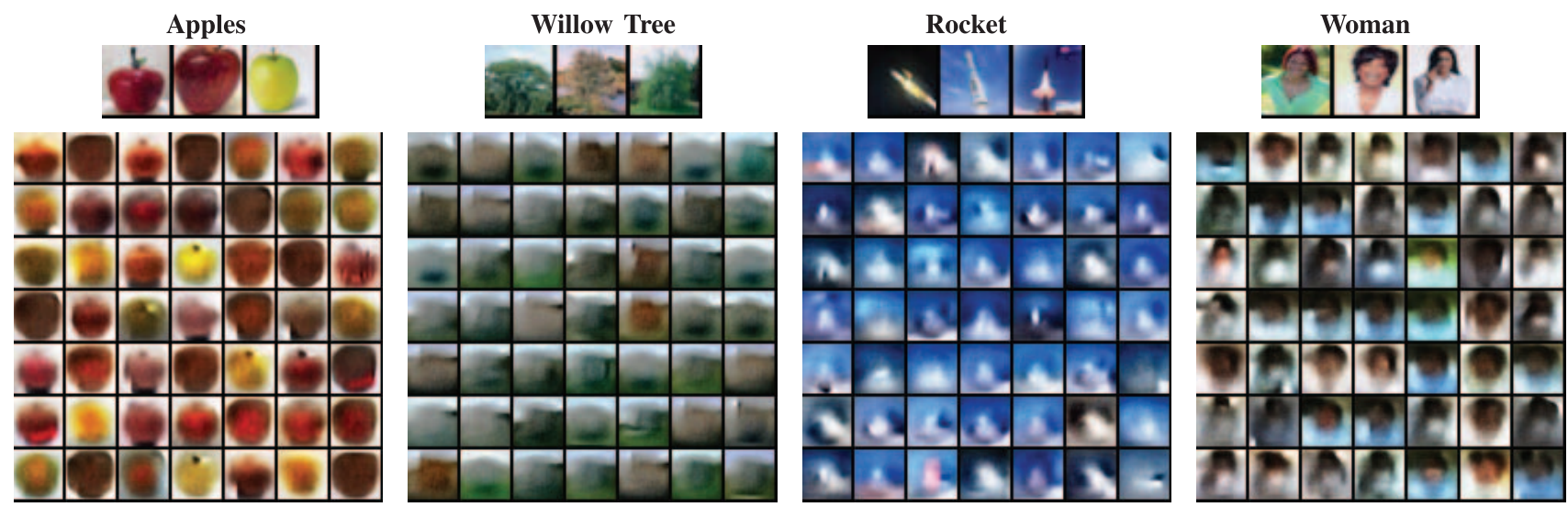

Fig. 7. Conditional samples generated by the HDP-DBM model when learning with only three training examples of a novel class: Top: Three training examples, Bottom: 49 conditional samples. Best viewed in color.

Fig. 9 displays a random subset of training images, along with the first and second layer DBM features, as well as

\section{Learning with 3 examples}
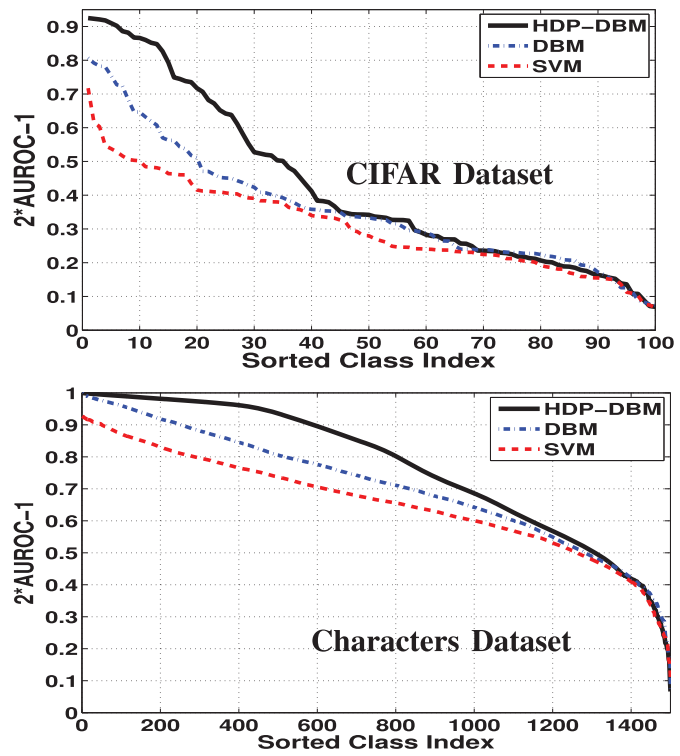

Fig. 8. Performance of HDP-DBM, DBM, and SVMs for all object classes when learning with three examples. Object categories are sorted by their performance. higher level class-sensitive HDP features. The first layer features capture low-level features, such as edges and corners, while the HDP features tend to capture higher level parts, many of which resemble pen "strokes," which is believed to be a promising way to represent characters [18]. The model discovers approximately 50 supercategories, and Fig. 10 shows a typical partition of some of the classes into supercategories which share the same prior distribution over "strokes." Similarly to the CIFAR dataset, many of the supercategories contain meaningful groups of characters.

Table 1 further shows results for classifying 15,000 test images as belonging to the novel versus all of the other 1,499 character classes. The results are averaged over 200 characters chosen at random, using the "leave-oneout" test format. The HDP-DBM model significantly outperforms other methods, particularly when learning characters with few training examples. This result demonstrates that the HDP-DBM model is able to successfully transfer appropriate prior over higher level "strokes" from previously learned categories.

We next tested the generative aspect the HDP-DBM model. Fig. 11 displays learned superclasses along with examples of entirely novel characters that have been generated by the model for the same superclass. In particular, the left panels show training characters in one supercategory with each row displaying a different observed character and each column displaying a drawing 


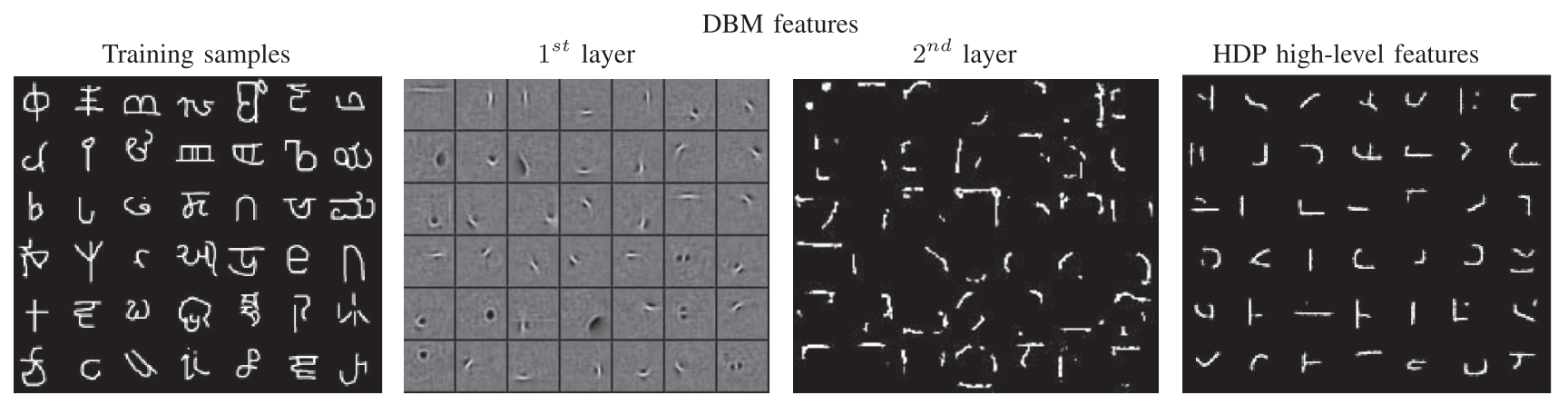

Fig. 9. A random subset of the training images along with the first and second layer DBM features, as well as higher level class-sensitive HDP features/topics. To visualize higher level features, we first sample M words from a fixed topic $\phi_{t}$, followed by sampling pixel values from the conditional DBM model.

produced by a different subject. The right panels show examples of novel synthesized characters in the corresponding supercategory, where each row displays a different synthesized character, whereas each column shows a different example generated at random by the HDP-DBM model. Note that many samples look realistic, containing coherent, long-range structure, while at the same time being different from existing training images.

Fig. 12 further shows conditional samples when learning with only three training examples of a novel character. Each panel shows three figures: 1) three training examples of a novel character class, 2) 12 synthesized examples of that class, and 3) samples of the training characters in the same supercategory that the novel character has been grouped under. Many of the novel characters are grouped together with related classes, allowing each character to inherit the prior distribution over similar high-level "strokes," and hence generalizing better to new instances of the corresponding class (see the supplemental materials, which can be found on the Computer Society Digital Library at http:/ / doi.ieeecomputersociety.org/10.1109/TPAMI.2012.269, for a much richer class of generated samples). Using DBNs instead of DBMs produced far inferior generative samples when generating new characters as well as when learning from three examples.

\subsection{Motion Capture}

Results on the CIFAR and Character datasets show that the HDP-DBM model can significantly outperform many other models on object and character recognition tasks. Features at all levels of the hierarchy were learned without assuming any image-specific priors, and the proposed model can be applied in a wide variety of application domains. In this section, we show that the HDP-DBM model can be applied to modeling human motion capture data.

The human motion capture dataset consists of sequences of 3D joint angles plus body orientation and translation, as shown in Fig. 13, and was preprocessed to be invariant to isometries [34]. The dataset contains 10 walking styles, including normal, drunk, graceful, gangly, sexy, dinosaur, chicken, old person, cat, and strong. There are 2,500 frames of each style at 60fps, where each time step was represented

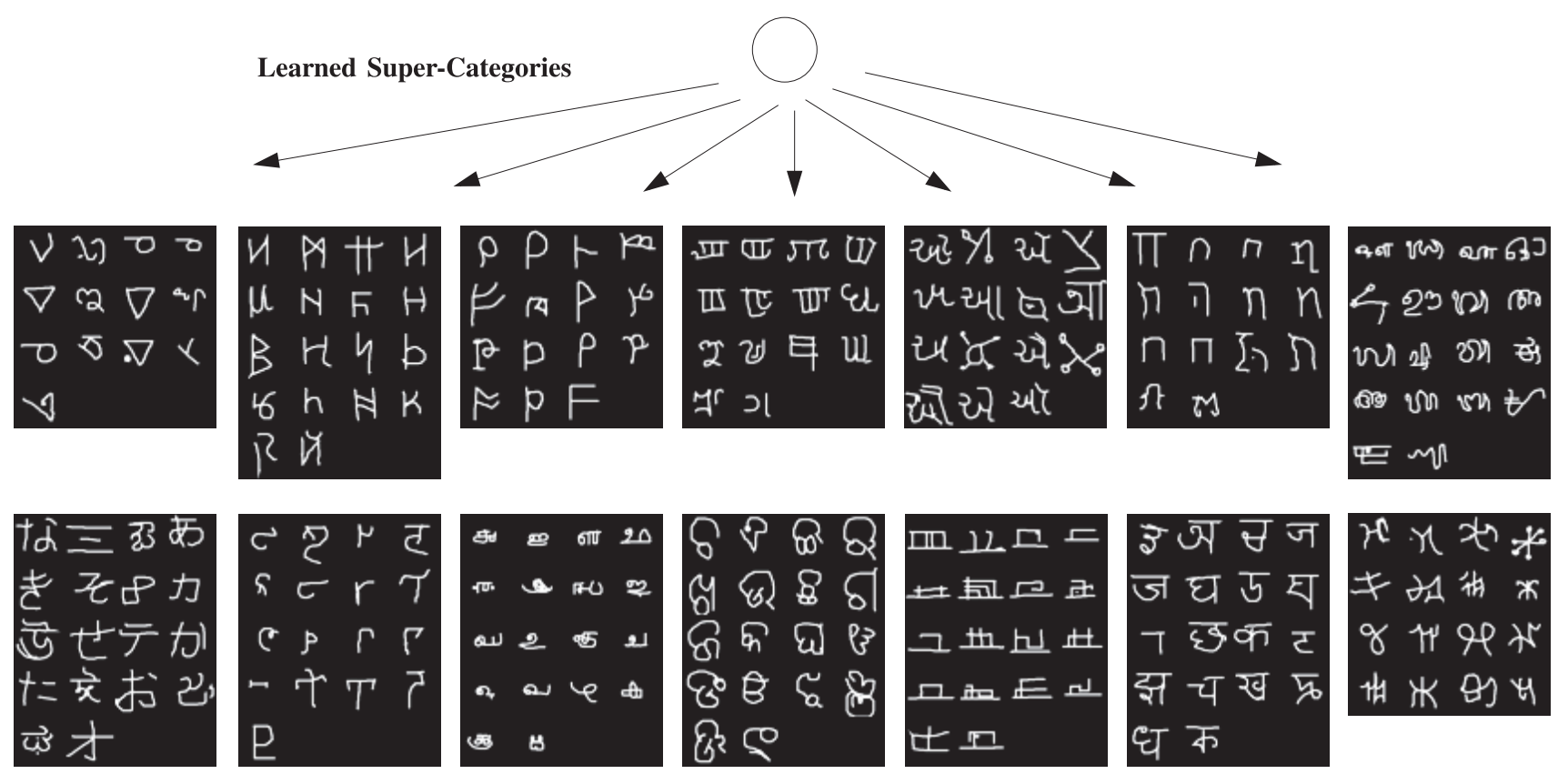

Fig. 10. Some of the learned supercategories that share the same prior distribution over "strokes." Many of the discovered supercategories contain meaningful groupings of characters. 


\section{Generated Samples}

\begin{tabular}{|c|c|c|c|c|c|}
\hline earne & Sampled characters & class & Sampled char & Learned super-class & Sampled characters \\
\hline$\checkmark v V$ & $\begin{array}{lllll}\lambda & 4 & ? & \lambda & \lambda\end{array}$ & $\mu$ & 111 & $\rho \rho \rho$ & $P$ \\
\hline ) 2.7 & 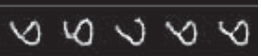 & & 44 & 巾 & $P P$ \\
\hline 0 & ष ए ए ৩ ए & า & $\| 11$ & 快 & H \\
\hline$\nabla \nabla \nabla$ & 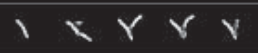 & i $P$ P & स भ भा भा सा & $p p p$ & P \\
\hline Y & $\nabla 55<5$ & $\mathrm{HH}$ & 人にん人 & $\approx \approx \approx \approx F F$ & $P P P 4 F$ \\
\hline 5 চ চ & $\leftarrow \kappa \tau \alpha \tau$ & $\breve{U} \bar{N}$ & $P . P P . P$. & $r r+r$ & $p$ \\
\hline \% & $\pi \pi \pi$ & & 14 & $F F F$ & $r$ \\
\hline इ ड & \& & & $\mathrm{T}$ & म म & ण ग \\
\hline जाघ छ छ & ता ता हा ता & & า า & Л лл Л & $\tau$ \\
\hline इ इ इ इ Б & ৫ ர৫ে ৫ & $\Gamma$ & 7 & ज $\bar{r}$ ज & $\pi$ त \\
\hline ख ख य ख ख & घ व व ब्र ब & 5 & $\pi \pi \pi$ & えをてたえ & r \\
\hline उ 3 उ 3 & $r d x$ of $\pi$ r & 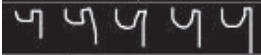 & $\gamma$ ir $\gamma$ & 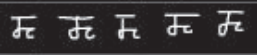 & - ta par m \\
\hline इक द छ ह & ন ब ন ন & या पा पा य & フ曰 $\triangle$ 口 & ग ग ग & $\pi_{0} \bar{r}_{0}$ \\
\hline & व च प्छ $\bar{~}$ & $h \quad 4$ & 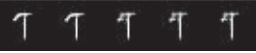 & ग ग ग & $<$ \\
\hline
\end{tabular}

Fig. 11. Within each panel: Left: Examples of training characters in one supercategory: Each row is a different training character and each column is a drawing produced by a different subject. Right: Examples of novel sampled characters in the corresponding supercategory: Each row is a different sampled character, and each column is a different example generated at random by the model.

Learning with Three Examples
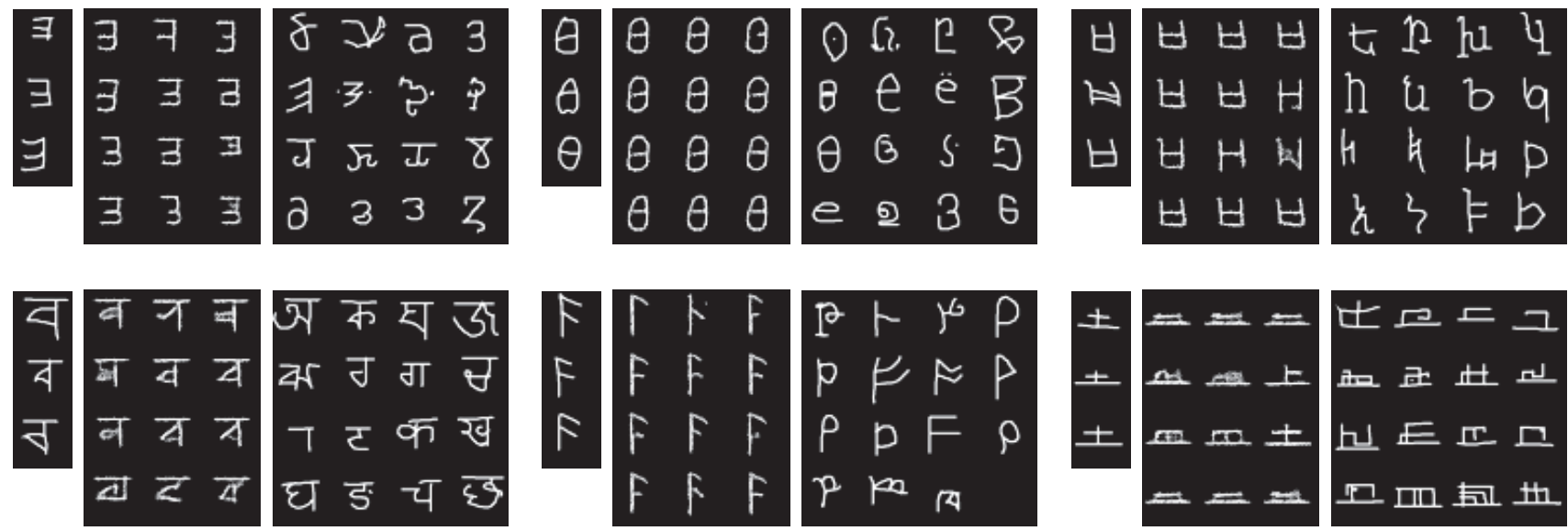

Fig. 12. Each panel shows three figures from left to right: 1) three training examples of a novel character class, 2) 12 synthesized examples of that class, and 3) training characters in the same supercategory that the novel character has been assigned to.

by a vector of 58 real-valued numbers. The dataset was split at random into 1,500 training and 1,000 test frames of each style. We further preprocessed the data by treating each window of 10 consecutive frames as a single $58 * 10=580$-d data vector.

For the two-layer DBM model, the first layer contained 500 hidden units, with the second layer containing $M=50$ softmax units. The HDP prior over the second-layer features was fit to various walking styles. Using "leaveone-out" test format, Table 1 shows that the HDP-DBM model performs much better compared to other models when discriminating between existing nine walking styles versus novel walking style. The difference is particularly large in the regime when we observe only a handful of training examples of a novel walking style.

\section{Conclusions}

We developed a compositional architecture that learns an HDP prior over the activities of top-level features of the DBM model. The resulting compound HDP-DBM model is able to learn low-level features from raw, high-dimensional sensory input, high-level features, as well as a category hierarchy for parameter sharing. Our experimental results show that the proposed model can acquire new concepts from very few examples in a diverse set of application domains.

The compositional model considered in this paper was directly inspired by the architecture of the DBM and HDP, but it need not be. Indeed, any other deep learning module, including DBNs, sparse autoencoders, or any other $\mathrm{HB}$ model, can be adapted. This perspective opens a space of 

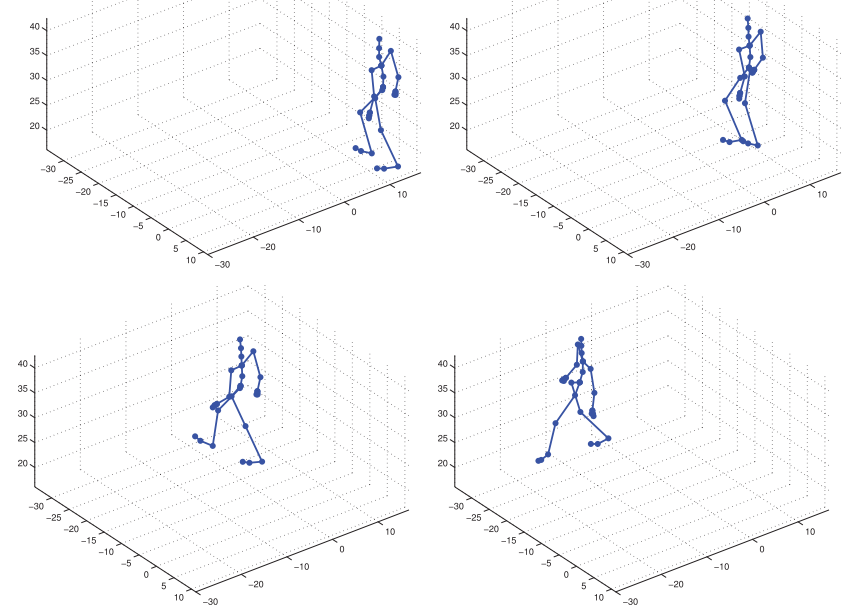

Fig. 13. Human motion capture data that corresponds to the "normal" walking style.

compositional models that may be more suitable for capturing the human-like ability to learn from few examples.

\section{ACKNOWLEDGMENTS}

This research was supported by NSERC, ONR (MURI Grant 1015GNA126), ONR N00014-07-1-0937, ARO W911NF-08-10242, and Qualcomm.

\section{REFERENCES}

[1] B. Babenko, S. Branson, and S.J. Belongie, "Similarity Functions for Categorization: From Monolithic to Category Specific," Proc. IEEE Int'l Conf. Computer Vision, 2009.

[2] E. Bart, I. Porteous, P. Perona, and M. Welling, "Unsupervised Learning of Visual Taxonomies," Proc. IEEE Conf. Computer Vision and Pattern Recognition, pp. 1-8, 2008.

[3] E. Bart and S. Ullman, "Cross-Generalization: Learning Novel Classes from a Single Example by Feature Replacement," Proc. IEEE Conf. Computer Vision and Pattern Recognition, pp. 672-679, 2005.

[4] D.M. Blei, A.Y. Ng, and M.I. Jordan, "Latent Dirichlet Allocation," J. Machine Learning Research, vol. 3, pp. 993-1022, 2003.

[5] D.M. Blei, T.L. Griffiths, and M.I. Jordan, "The Nested Chinese Restaurant Process and Bayesian Nonparametric Inference of Topic Hierarchies," J. ACM, vol. 57, no. 2, 2010.

[6] K.R. Canini and T.L. Griffiths, "Modeling Human Transfer Learning with the Hierarchical Dirichlet Process," Proc. NIPS Workshop Nonparametric Bayes, 2009.

[7] C.-C. Chang and C.-J. Lin, "LIBSVM: A Library for Support Vector Machines," ACM Trans. Intelligent Systems and Technology, vol. 2, pp. 27:1-27:27, 2011.

[8] B. Chen, G. Polatkan, G. Sapiro, D.B. Dunson, and L. Carin, "The Hierarchical Beta Process for Convolutional Factor Analysis and Deep Learning," Proc. 28th Int'l Conf. Machine Learning, pp. 361368,2011

[9] A. Coates, B. Carpenter, C. Case, S. Satheesh, B. Suresh, T. Wang, and A.Y. Ng, "Text Detection and Character Recognition in Scene Images with Unsupervised Feature Learning," Proc. 11th Int'l Conf. Document Analysis and Recognition, 2011.

[10] A. Courville, J. Bergstra, and Y. Bengio, "Unsupervised Models of Images by Spike-and-Slab RBNS," Proc. 28th Int'l Conf. Machine Learning, pp. 1145-1152, June 2011.

[11] L. Fei-Fei, R. Fergus, and P. Perona, "One-Shot Learning of Object Categories," IEEE Trans. Pattern Analysis and Machine Intelligence, vol. 28, no. 4, pp. 594-611, Apr. 2006.

[12] G.E. Hinton, S. Osindero, and Y.2. Teh, "A Fast Learning Algorithm for Deep Belief Nets," Neural Computation, vol. 18, no. 7, pp. 1527-1554, 2006.
[13] G.E. Hinton and R.R. Salakhutdinov, "Reducing the Dimensionality of Data with Neural Networks," Science, vol. 313, no. 5786 , pp. $504-507,2006$

[14] G.E. Hinton and T. Sejnowski, "Optimal Perceptual Inference," Proc. IEEE Conf. Computer Vision and Pattern Recognition, 1983.

[15] C. Kemp, A. Perfors, and J. Tenenbaum, "Learning Overhypotheses with Hierarchical Bayesian Models," Developmental Science, vol. 10, no. 3, pp. 307-321, 2006.

[16] A. Krizhevsky, "Learning Multiple Layers of Features from Tiny Images," 2009

[17] A. Krizhevsky, "Learning Multiple Layers of Features from Tiny Images," technical report, Dept. of Computer Science, Univ. of Toronto, 2009.

[18] B. Lake, R. Salakhutdinov, J. Gross, and J. Tenenbaum, "One-Shot Learning of Simple Visual Concepts," Proc. 33rd Ann. Conf. Cognitive Science Soc., 2011.

[19] H. Larochelle, Y. Bengio, J. Louradour, and P. Lamblin, "Exploring Strategies for Training Deep Neural Networks." J. Machine Learning Research, vol. 10, pp. 1-40, 2009.

[20] H. Lee, R. Grosse, R. Ranganath, and A.Y. Ng, "Convolutional Deep Belief Networks for Scalable Unsupervised Learning of Hierarchical Representations," Proc. Int'l Conf. Machine Learning, pp. 609-616, 2009.

[21] H. Lee, R. Grosse, R. Ranganath, and A.Y. Ng, "Convolutional Deep Belief Networks for Scalable Unsupervised Learning of Hierarchical Representations," Proc. 26th Int'l Conf. Machine Learning, pp. 609-616, 2009.

[22] Y. Lin, T. Zhangi, S. Zhu, and K. Yu, "Deep Coding Networks," Proc. Advances in Neural Information Processing Systems Conf., vol. 23, 2011.

[23] A. Mohamed, G. Dahl, and G. Hinton, "Acoustic Modeling Using Deep Belief Networks," IEEE Trans. Audio, Speech, and Language Processing, vol. 20, no. 1, pp. 14-22, Jan. 2012.

[24] V. Nair and G.E. Hinton, "Implicit Mixtures of Restricted Boltzmann Machines," Proc. Advances in Neural Information Processing Systems Conf., vol. 21, 2009.

[25] A. Perfors and J.B. Tenenbaum, "Learning to Learn Categories," Proc. 31st Ann. Conf. Cognitive Science Soc., pp. 136-141, 2009.

[26] M.A. Ranzato, Y. Boureau, and Y. LeCun, "Sparse Feature Learning for Deep Belief Networks," Proc. Advances in Neural Information Processing Systems, 2008.

[27] H. Robbins and S. Monro, "A Stochastic Approximation Method," Annals Math. Statistics, vol. 22, pp. 400-407, 1951.

[28] A. Rodriguez, D. Dunson, and A. Gelfand, "The Nested Dirichlet Process." J. Am. Statistical Assoc., vol. 103, pp. 1131-1144, 2008.

[29] R.R. Salakhutdinov and G.E. Hinton, "Deep Boltzmann Machines," Proc. Int'l Conf. Artificial Intelligence and Statistics, vol. 12, 2009.

[30] R.R. Salakhutdinov and G.E. Hinton, "Replicated Softmax: An Undirected Topic Model," Proc. Advances in Neural Information Processing Systems Conf., vol. 22, 2010.

[31] L.B. Smith, S.S. Jones, B. Landau, L. Gershkoff-Stowe, and L. Samuelson, "Object Name Learning Provides On-the-Job Training for Attention," Psychological Science, vol. 13, pp. 13-19, 2002.

[32] R. Socher, C. Lin, A.Y. Ng, and C. Manning, "Parsing Natural Scenes and Natural Language with Recursive Neural Networks," Proc. 28th Int'l Conf. Machine Learning, 2011.

[33] E.B. Sudderth, A. Torralba, W.T. Freeman, and A.S. Willsky, "Describing Visual Scenes Using Transformed Objects and Parts," Int'l J. Computer Vision, vol. 77, nos. 1-3, pp. 291-330, 2008.

[34] G. Taylor, G.E. Hinton, and S.T. Roweis, "Modeling Human Motion Using Binary Latent Variables," Proc. Advances in Neural Information Processing Systems Conf., 2006.

[35] G.W. Taylor, R. Fergus, Y. LeCun, and C. Bregler, "Convolutional Learning of Spatio-Temporal Features," Proc. 11th European Conf. Computer Vision, 2010.

[36] Y.W. Teh and G.E. Hinton, "Rate-Coded Restricted Boltzmann Machines for Face Recognition," Proc. Advances in Neural Information Processing Systems Conf., vol. 13, 2001.

[37] Y.W. Teh and M.I. Jordan, "Hierarchical Bayesian Nonparametric Models with Applications," Bayesian Nonparametrics: Principles and Practice, Cambridge Univ. Press, 2010.

[38] Y.W. Teh, M.I. Jordan, M.J. Beal, and D.M. Blei, "Hierarchical Dirichlet Processes," J. Am. Statistical Assoc., vol. 101, no. 476, pp. 1566-1581, 2006. 
[39] T. Tieleman, "Training Restricted Boltzmann Machines Using Approximations to the Likelihood Gradient," Proc. 25th Int'l Conf. Machine Learning, 2008.

[40] A. Torralba, R. Fergus, and W.T. Freeman, "80 Million Tiny Images: A Large Data Set for Non-Parametric Object and Scene Recognition," IEEE Trans. Pattern Analysis and Machine Intelligence, vol. 30, no. 11, pp. 1958-1970, Nov. 2008.

[41] A. Torralba, R. Fergus, and Y. Weiss, "Small Codes and Large Image Databases for Recognition," Proc. IEEE Conf. Computer Vision and Pattern Recognition, 2008.

[42] A.B Torralba, K.P Murphy, and W.T. Freeman, "Shared Features for Multiclass Object Detection," Toward Category-Level Object Recognition, pp. 345-361, 2006.

[43] P. Vincent, H. Larochelle, Y. Bengio, and P. Manzagol, "Extracting and Composing Robust Features with Denoising Autoencoders," Proc. 25th Int'l Conf. Machine Learning, vol. 307, pp. 1096-1103, 2008.

[44] F. Xu and J.B. Tenenbaum, "Word Learning as Bayesian Inference," Psychological Rev., vol. 114, no. 2, pp. 245-272, 2007.

[45] L. Younes, "Parameter Inference for Imperfectly Observed Gibbsian Fields," Probability Theory Related Fields, vol. 82, pp. 625-645, 1989.

[46] L. Younes, "On the Convergence of Markovian Stochastic Algorithms with Rapidly Decreasing Ergodicity Rates," Mar. 2000.

[47] A.L. Yuille, "The Convergence of Contrastive Divergences," Proc. Advances in Neural Information Processing Systems Conf., 2004.

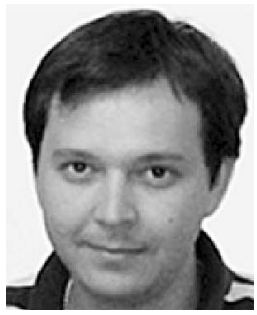

Ruslan Salakhutdinov received the $\mathrm{PhD}$ degree in machine learning (computer science) from the University of Toronto, Ontario, Canada, in 2009. After spending two postdoctoral years at the Massachusetts Institute of Technology Artificial Intelligence Lab, he joined the University of Toronto as an assistant professor in the Departments of Statistics and Computer Science. His primary interests lie in artificial intelligence, machine learning, deep learning, and large-scale optimization. He is and Alfred P. Sloan Research Fellow and Microsoft Research New Faculty Fellow, a recipient of the Early Researcher Award, Connaught New Researcher Award, and is a Scholar of the Canadian Institute for Advanced Research.

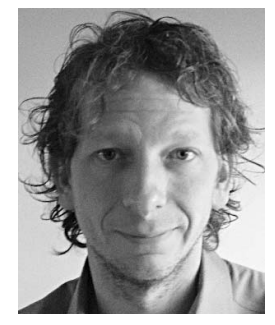

Joshua B. Tenenbaum received the $\mathrm{PhD}$ degree in the Department of Brain and Cognitive Sciences, Massachusetts Institute of Technology, Cambridge, in 1993, where he is currently a professor of computational cognitive science as well as a principal investigator in the Computer Science and Artificial Intelligence Laboratory. He studies learning, reasoning, and perception in humans and machines, with the twin goals of understanding human intelligence in computational terms and bringing computers closer to human capacities. He and his collaborators have pioneered accounts of human cognition based on sophisticated probabilistic models and developed several novel machine learning algorithms inspired by human learning, most notably Isomap, an approach to unsupervised learning of nonlinear manifolds in highdimensional data. His current work focuses on understanding how people come to be able to learn new concepts from very sparse data-how we "learn to learn"-and on characterizing the nature and origins of people's intuitive theories about the physical and social worlds. His papers have received awards at the IEEE Computer Vision and Pattern Recognition (CVPR), NIPS, Cognitive Science, UAI, and IJCAI conferences. He is the recipient of early career awards from the Society for Mathematical Psychology, the Society of Experimental Psychologists, and the American Psychological Association, along with the Troland Research Award from the National Academy of Sciences.

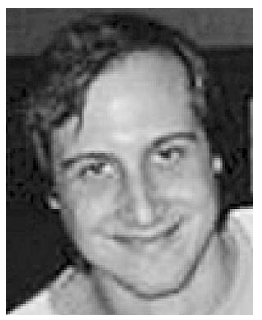

Antonio Torralba received the degree in telecommunications engineering from the Universidad Politecnica de Cataluna, Barcelona, Spain, and the $\mathrm{PhD}$ degree in signal, image, and speech processing from the Institute National Polytechnique de Grenoble, France. Thereafter he spent postdoctoral training at the Brain and Cognitive Science Department and the Computer Science and Artificial Intelligence Laboratory at the Massachusetts Institute of Technology (MIT), Cambridge. He is an associate professor of electrical engineering and computer science in the Computer Science and Artificial Intelligence Laboratory at MIT. He is a member of the IEEE.

$>$ For more information on this or any other computing topic, please visit our Digital Library at www.computer.org/publications/dlib. 